\title{
Establishing a Holy Lineage: Theodore the Stoudite's Funerary Catechism for His Mother (BHG 2422)
}

\author{
STEPHANOS EFTHYMiAdis / J. M. FEATHERSTONE
}

Introduction, edition and annotation (Stephanos Efthymiadis)

Though the same interests, themes and issues run through his prolific work, of all writers of ninth- and tenth-century Byzantium Theodoros Stoudites stands out as the most original and versatile in terms of language, style and literary genre. His diction mostly combined ecclesiastical and sometimes demotic Greek with the new vocabulary of an inspired craftsman of language; his usually straightforward prose style at times betrayed sophistication and a learned background corresponding to the social status and education of his addressee or honoree; finally, whether in poetry or in prose, his selection of literary genre ranged from the iambic twelve-syllable verse to the Catechism and the Funeral Oration, forms with no recent recorded precedent. By and large, this variety was the natural result of a multifaceted personality engaged in an ongoing struggle in defense and promotion of both public and private interests. The modification of language and style and shifting of genres were prompted by a recurrent and renewable involvement of himself and his monastic community in such crises as the Moechian controversy and Second Iconoclasm. Above all, however, in his writings Theodoros was prudent enough both to blur and draw the dividing line between introversion and extroversion, making things private appear public and vice versa.

In the present study we shall deal with one of his earliest texts which enshrines both the public and the private, the Funeral Catechism for his mother Theoktiste (BHG 2422). ${ }^{1}$ The appreciable proportion of seventy-six letters addressed to women and included in his copious correspondence, available in the admirable critical edition of Georgios Fatouros, shows self-asserting Theodoros' openness to the other half of human kind, a feature seldom encountered in the writings of a monastic father. Arguably, this concern was prompted by his close

1 For a detailed biographical sketch of Theoktiste see PmbZ 8032; and PBE 1: Theoktiste 3. As one of his well-known works the Catechism was cited in the vita of Theodoros Stoudites A (BHG 1754), in: PG 99, col. 117A; vita B (BHG 1755), ibid., col. 237A; and vita C (BHG 1755d), ed. V. Latyšev, in: VV 21 (1914) 259-260. 
links to Constantinopolitan aristocracy and its officialdom as well as his endeavour to reinforce right faith and discipline in an age marked by doctrinal and spiritual temptations. ${ }^{2}$

It is among the first letters of this correspondence that we find his first extant

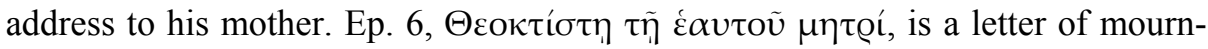
ing, penned in a state of poignant sorrow, not long before her death and after the premature loss of his not named sister and his brother Euthymios. ${ }^{3}$ In this lamentation Theoktiste is repeatedly styled as a saint who rejected mundane glory for the heritage of heaven, who shared the exploits of martyrdom though not shedding blood, who was bereft of her limbs, i.e., her children, for the love of God. ${ }^{4}$

Apart from this letter, Theodoros addressed his mother after her death in a

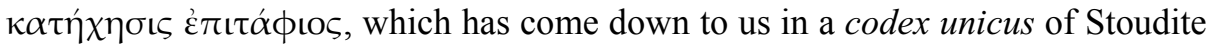
provenance, Parisinus graecus 1491 (siglum P). This is a parchment, 24 x 37,7 $\mathrm{cm}$, written in two columns at the beginning of the 10th century by two scribes; the first copied ff. $1-198^{\mathrm{v}}$ (36 lines to page), whereas the second copied ff. 199$245^{\mathrm{v}}$ (36-39 lines to page). ${ }^{5}$ The Catechism is contained in ff. $94-103$ and it is transmitted in good shape with only a few spelling errors; it ends rather abruptly in the second line of f. 103 and is followed by a lengthy fragment of an untitled text given in the form of "Question and Answer." This text has been identified with the Oratio de theatris et de Abraham, a spurious work of St John Chrysostom, oddly copied in this part of the manuscript. ${ }^{6}$

The fact that the Catechism for his mother was included in a hagiographical collection produced in the monastery of Stoudios provides sufficient proof that,

2 For these letters see J. Gouillard, La femme de qualité dans les lettres de Théodore Studite, in: JÖB 32/2 (1982) 445-453; and P. Hatlie, Women of Discipline During the Iconoclast Age, in: BZ 89 (1996) 37-44.

3 Theodori Studitae epistulae, ed. G. Fatouros (CFHB XXXI/1-2 - Series Berolinensis), Berlin/NewYork 1992, 21-23; Theodoros is also alluding twice to the loss of his siblings in vv. 9 10 and 24-25. On the same letter see A. P. Dobroklonskij, Преподовний Феодор исповедник и игумен Студийски, Odessa 1913, I 295-298; also Fatouros, ibid., 148*-149*; and V. Sarris,

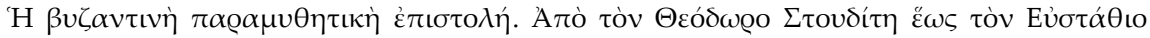

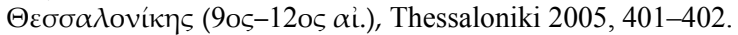

4 For Theodoros' idea that people of his era could emulate the deeds of the early Christian martyrs see P. Hatlie, The Politics of Salvation: Theodore of Stoudios on Martyrdom (Martyrion) and Speaking Out (Parrhesia), in: DOP 50 (1990) 266-272.

5 Contents of the manuscript are given in H. Omont, Inventaire sommaire des manuscrits grecs de la Bibliothèque Nationale, Paris 1898, II 64; F. Halkin, Manuscrits grecs de Paris: Inventaire hagiographique (SubHag 44), Brussels 1968, 182-183. On other earlier manuscripts of Stoudite origin see N. F. Kavrus, Студийский скрипторий в IX в. (по материалам рукопишей Москвы и Ленинграда), in: VV 44 (1983) 98-110.

6 See Bibliotheca Hagiographica Graeca, Novum Auctarium (SubHag 65), Brussels 1984, nos $2349 t$ and 2355. The fragment corresponds to PG 56, cols 543-554. Theodori Studitae epistulae, ed. Fatouros (see n. 3), 28* has taken it as an unpublished work of Theodoros on the Sunday of Forefathers. 
together with his letter, this Funeral Catechism aimed at propounding Theoktiste's holy commemoration. ${ }^{7}$ Before being reprinted in Migne's Patrologia Graeca with some typing errors, this text was edited by A. Mai in his Nova patrum bibliotheca. ${ }^{8}$ Since this edition is not free of transcription errors and omissions, we deemed it worthy to offer a new one followed by an English translation and succinct commentary given in the form of notes. ${ }^{9}$

Judging from the fact that Theoktiste's death is presented as imminent and irreversible, it is plausible to assume that only a short time separated the composition of Theodoros' Catechism from that of his letter. The Catechism has reasonably been dated to the period between 797 and 802, i.e., during Eirene's reign as a

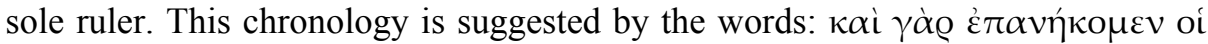

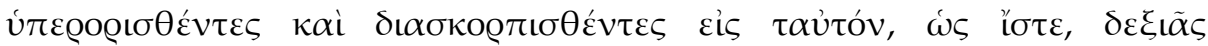

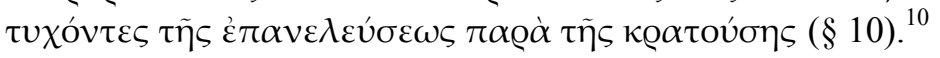

Whether departing from historical considerations or not, older and recent surveys of the literary output either of the iconoclastic period in general or Theodoros Stoudites in particular, have not done full justice to this text, perhaps on the single grounds that it was not ranked among his most important literary creations. However, it is no exaggeration to say that, by virtue of the date of its composition alone, the Funeral Catechism for Theoktiste represents both a starting point for its author and a turning one in female sainthood and hagiography. As it will be argued, by launching, on the one hand, a new model of female sainthood - that of a pious housewife ending her marriage and entering the monastery, it drastically parted from the Late Antique past and foreshadowed later medieval examples; and by sketching, on the other hand, a sacred portrait of a mother, it paved the son's way towards holiness.

From Charles Diehl to Alexander Kazhdan scholars have outlined this text's idiosyncratic features as regards both the portrayal of the heroine and the highlights of her life upon it called attention: Theoktiste's tonsure and her overcoming

7 Details in F. Halkin, La Passion de Sainte Théoctiste, in: AB 73 (1955) 55 (= Martyrs Grecs IIe-VIIIe s. [Variorum Collected Studies Series 30], London 1974, II).

8 See Nova patrum bibliotheca, VI/2, Rome 1854, 364-378; and as Oratio 13 in: PG 99, cols 884 902.

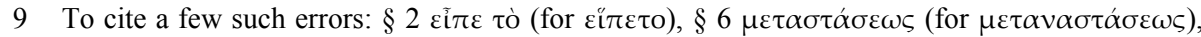

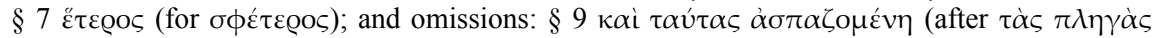

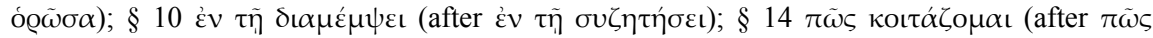
$\dot{\alpha} v i ́ \sigma \tau \alpha \mu \alpha \mathrm{l})$.

10 Conversely, erroneously considering that Theoktiste outlived her brother Plato, A. Sideras dated it to ca. 820; see his Die byzantinischen Grabreden (WBS XIX), Vienna 1994, 99-100. Long before him, B. Hermann considered that in 807-808 Theoktiste was still alive: see Theoktista von Byzanz, die Mutter zweier Heiliger, Freiburg 1919, 108ff. Theodoros simply hinted at his deceased mother in a letter that he addressed to the nun Anna in ca. 809-811, i.e., later than this period; see Theodori Studitae epistulae (see n. 3), 42,2-3, 122. 
of maternal sentiments; the relationship of mother and son as well as their separation; the spiritual advancement of a woman full of energy and strength who, both in the world and the monastery, could, at instances, be overbearing to people under her charge. The historian Kazhdan went on to underscore that, unlike the Funeral Laudation to his uncle Plato, this funeral sermon was not "historical" and "eventful"; and, what is more, it lacked allusions to the question and the cult of icons. ${ }^{11}$ As a matter of fact, composed in the interim period between the two Iconoclasms, just ten years after the Council of Nicaea II (787), this oration is a text poor in ecclesiastical polemic concerning Iconoclasm. Just once in his narra-

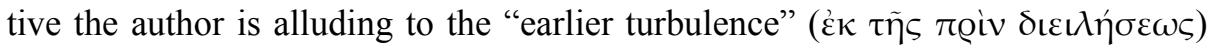
that had spoiled monasticism $(\S 8)$.

Though posed as a riddle by Kazhdan, several reasons can be adduced to account for this silence on icon-worship. First and foremost, Theoktiste the woman could not claim any direct (and public) involvement in the anti-iconoclastic struggle, nor her son at that point. ${ }^{12}$ Besides that, in the Laudation of his uncle Plato, composed much later, in ca. 814, we hear much about First Iconoclasm but nothing of any family resistance. Moreover, the reign of the "much loathsome" Constantine V saw Plato not only recovering from the shock of the plague of 747-748 which had his parents as victims but also, as a notarios, winning fortune and reputation in the imperial quarters. ${ }^{13}$ The "pressing issue" at the time when Theodoros delivered his Catechism was the Moechian controversy, then in its first phase. This impression is further borne out by his first five letters of the collection which date from the same period 795-797 (epp. 1-5). Much more than Iconoclasm, Theodoros' sermon had good reasons to concentrate on the hardships that he and his uncle Plato endured on account of their opposition to the "adulterous marriage" of Constantine VI, to which the patriarch Tarasios offered his silent support. ${ }^{14}$ Naturally, with her brother and son recently recalled from exile by the

11 See Ch. Diehl, Une bourgeoise de Byzance au VIIIe siècle, in: Figures Byzantines, Paris 1906, I 111-132 ; and A. Kazhdan, A History of Byzantine Literature (650-850). In collaboration with L. F. Sherry/Ch. Angelidi, Athens 1999, 244-247. The text was also included in D. L. Za-

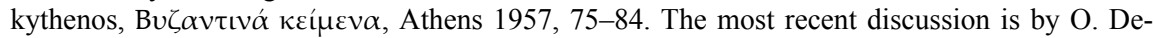
louis, Saint Jean-Baptiste de Stoudios à Constantinople. La contribution d'un monastère à l'histoire de l'Empire byzantin (v. 454-1204), Thèse présentée pour l'obtention du grade de Docteur en l'Histoire de l'Université Paris I-Panthéon Sorbonne, Paris 2005, 308-309.

12 Naturally enough, Theoktiste was excluded from A. Kazhdan's and A.-M. Talbot's survey of the role of women in Iconoclasm, see: Women and Iconoclasm, in: BZ 84/85 (1991-1992) 391408.

13 See Laudatio Platonis, in: PG 99, col. 808A-B.

14 Along with her relatives, Theoktiste is said to have suffered a thirty-day imprisonment ( $§ 10)$. For a commentary on these allusions see E. von Dobschütz, Methodius und die Studiten, in: BZ 18 (1909) 60. 
empress Eirene, Theoktiste was crowned with a resistance which was monopolized by her family and her monastic milieu.

Nonetheless, the Funeral Catechism for his mother and the Laudation to his uncle diverge not only on the question of Iconoclasm but on matters of literary form and orientation. Thomas Pratsch singled out the topoi of piety, humility, disruption of blood bonds and other things, upon which Theodoros canvassed his mother's sainthood in his Catechism. ${ }^{15}$ This, however, should not leave the impression that Theodoros' sermon adheres to the laws of hagiographical praise at the expense of recording real life. On the contrary, Theodoros considerably distances himself from the hagiographical stereotypes: idealized homeland, parenthood and childhood are passed over in silence and so is marital engagement. ${ }^{16}$ Details on these matters can be drawn only from the Laudation to Plato, which, as already noted, is much more concerned with historicity than the sermon pronounced in honour of Theoktiste; the latter was based more on situations and scenes taken from the real life, all of which stand for eye-witness reports, rather than being modeled upon the typical patterns of a saint's Life. In other words, although the argument and the purpose of the praise to his mother were ultimately hagiographic, the work itself, set and narrated by her son in a realistic fashion, is not hagiography in its common use and sense.

Following the sad announcement of her death in the preamble, Theodoros avoids clinging to the picture of an "ideal passive heroine" whose holiness must appear prefigured. Instead of attaching to her the stereotypes of a noble origin and a prudent childhood, Theodoros portrays his mother as a forceful personality who imposes her own model of holy life and conduct on her family milieu and beyond. Unlike iconophile male and female saints whose holy portrait was mostly derived from hagiographical re-adaptations of historical reality, Theoktiste is depicted as a woman in flesh and blood, with a temperament pairing philanthropy with behaviours not se ipso rational. Though translating her violent outbreaks as ultimately leading to repentance, her son does not refrain from divulging them in two instances, while treating her life in family and in the monastery ( $\S \S 5$ and 12$)$.

15 Th. Pratsch, Theodoros Studites (759-826) - zwischen Dogma und Pragma. Der Abt des Studiosklosters in Konstantinopel im Spannungsfeld von Patriarch, Kaiser und eigenem Anspruch (BBS 4), Frankfurt am Main 1998, 29-33; and idem, Der hagiographische Topos. Griechische Heiligenviten in mittelbyzantinischer Zeit (Millennium Studien 6), Berlin/New York 2005, 51, 96, 212 (Weitere Tugenden); 329 (Verteilung der Habe); 331 (Letzte Amtshandlungen).

16 Among the hagiography of the period similar glossing over the topoi of homeland, parents and childhood is observable in the Life of St Euthymios of Sardis, a work penned in 832 by the future patriarch Methodios. Yet, this "omission" is not accounted for on the simple grounds of ignorance but on the awareness that none of this is necessarily conducive to virtue; see J. Gouillard, La vie d'Euthyme de Sardes († 831). Une œuvre du patriarche Méthode, in: TM 10 (1987) $21-23(\S 2)$. 
Both the letter and the Catechism dedicated to Theoktiste constitute the earliest documented examples of portraying a holy woman in a new era, the Byzantine Middle Ages, and of family involvement in promoting the cult of a holy person. As has been pointed out, models of female sainthood were then inspired by the values of a new social elite and an emerging monastic culture. ${ }^{17}$ Family lineage gained large acclaim in society and lay behind the development of what has been termed "family cult". ${ }^{18}$ As can be traced in pieces of hagiography dating from the immediately following decades, this cult found fertile ground in monasteries founded or restored by members of the Constantinopolitan aristocracy within and outside the capital. ${ }^{19}$ Cases in point are the mother and the sister of St Stephanos the Younger whose vita was written in ca. 809 or the equally famous Philaretos the Merciful, praised by his grandson Niketas in ca. $822 .{ }^{20}$ On another level and beyond the confines of her age, dividing as she did her life between the marital and the monastic status, Theoktiste anticipated, to a great extent, the examples of holy women who attained sainthood either once they were widowed and entered a convent (Athanasia of Aegina, Theodora of Thessalonike) or without ever embracing monastic life (Thomaïs of Lesbos, Maria the Younger). Nevertheless, though a pious housewife practicing secret asceticism, Theoktiste neither "benefited" from her spouse's death nor became the wife-martyr suffering from a coarse and brutal husband. ${ }^{21}$ What is more, her piety was not vested with the usual colours of a passive humility and modesty, but with those of a woman tak-

17 See E. Patlagean, L'histoire de la femme déguisée en moine et l'évolution de la sainteté féminine à Byzance, in: SM, 3e série, XVII, Spoleto 1976, 617-623 (= Structure sociale, famille, chrétienté à Byzance. IVe-XIe siècle [Collected Studies Series 134], London 1981, XI).

18 I. Hausherr was the first to point out that the Stoudite's family was "une famille de saints"; see Le moine et l'amitié, in: Études de spiritualité orientale (OCA 183), Rome 1969, 338-340. Other examples from Middle Byzantine hagiography were discussed by A.-M. Talbot, Family Cults in Byzantium: the Case of St Theodora of Thessalonike, in: $\Lambda$ EIM $\Omega N$. Studies Presented to Lennart Rydén on his Sixty-fifth Birthday, ed. J. O. Rosenqvist, Uppsala 1996, 49-69 (= Women and Religious Life in Byzantium, Aldershot 2001, VI).

19 For the building activity of the period, especially that of the Stoudites, see V. Ruggieri, Byzantine Religious Architecture (582-867): Its History and Structural Elements (OCA 237), Rome 1991, 107-111.

20 See vita of St Stephen the Younger (BHG 1666), §§ 47 and 53, ed. M. F. Auzépy, La vie d'Étienne le Jeune par Étienne le Diacre (Birmingham Byzantine and Ottoman Monographs 3), Aldershot/Hampshire 1997, 148 and 153. Also vita of Philaretos the Merciful (BHG 1511z), ed. L. Rydén, The Life of St Philaretos the Merciful written by his Grandson Niketas (Acta Universitatis Upsaliensis. Studia Byzantina Upsaliensia 8), Uppsala 2002, 45-50.

21 For other similar examples of secret asceticism within marriage see D. de F. Abrahamse, Women's Monasticism in the Middle Byzantine Period: Problems and Prospects, in: BF 9 (1985) 53-54 and n. 53-54. For a recent innovative discussion of different types of female hagiography see S. Constantinou, Female Corporeal Performances. Reading the Body in Byzantine Passions and Lives of Holy Women (Acta Universitatis Upsaliensis. Studia Byzantina Upsaliensia 9), Uppsala 2005. 
ing action and gradually managing to impose her impulse towards monastic isolation on male and female members of her family. Granted, a few allusions in Theodoros' account allow us to suspect that her brother Plato played an influential role in this decision; ${ }^{22}$ yet, this fact alone does not diminish much of Theoktiste's consequential impact on driving, sooner or later, all members of her family (husband, sons and daughters) out of society. Unlike later examples from ninthand tenth-century hagiography where at least one member remains in the world to perpetuate the family line, in the case of Theoktiste rejection of the world has a total and overwhelming effect. ${ }^{23}$

Nonetheless, this is not the only feature that makes Theodoros' Catechism noteworthy; his text is perhaps unique in portraying a woman without interfering with gender issues. Although Theoktiste is not divested the "particulars" of a woman's life in society, references to inferiority and weakness as "befitting" female nature, yet surmounted by the engagement in a holy cause, or, to put it differently, a "gender-oriented" vocabulary is not what we encounter in her praise. Unlike Gorgonia, the married sister of Gregory of Nazianzos who also appropriated her husband to her pious way of life, Theoktiste's ascetical toils are not equated to those of men. Likewise, no matters of gender feature largely in the presentation of Theodoros' mother as they do in the philosophical biography of Gregory of Nyssa's sister, Macrina (d. 380) or in the Encomium which Michael Psellos wrote for his Mother. ${ }^{24}$ What is more, compared to them Theoktiste would appear superior in that she vanquished her illiteracy by learning the Psalter, a knowledge that she later passed on to her daughter. ${ }^{25}$ Notably, her son assigns this

22 Cf. $\S \S 6,8,9$. Their attraction to monastic vocation was credited to Plato also in the vitae of Theodoros: vitae A and B, in: PG 99, cols 121A-B and 240D-241A; vita C, ed. Latyšev (see n. 1), 261-262.

23 See the examples analyzed by Patlagean, L'histoire de la femme déguisée (see n. 17), 617-619. Theodoros highlights this lack in desire for the succession of the race by the words: ov $\tau \tilde{\omega}$

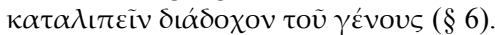

24 See De vita Macrinae, ch. 1, ed. P. Maraval, in : Grégoire de Nysse. Vie de Sainte Macrine (SC 178), Paris 1971, 140; cf. V. E. F. Harrison, Male and female in Cappadocian theology, in: JThS n.s. 41 (1996) 446-447. As for Psellos, he refers to his mother as a weak-natured person in two instances: "she was not a man by nature and was not thus allowed to study literature freely" and "she was one who knew nothing feminine, except what was decreed by nature, but was in all other respects strong and manly in soul and even showed herself to be more resilient than the other portion of our species" (U. Criscuolo, Autobiografia: Encomio per la sua madre [Speculum 11], Naples 1989, vv. 136-138 and 420-422, 90 and 99 respectively). Moreover, on another occasion, Psellos extols her mother for her mastery of nature and her reconciliation of feminine and masculine qualities (vv. 1595-1597, ibid., 141).

25 For a discussion see N. Kalogeras, The Role of Parents and Kin in the Education of Byzantine Children, in: Hoping for Continuity. Childhood, Education and Death in Antiquity and the Mid-

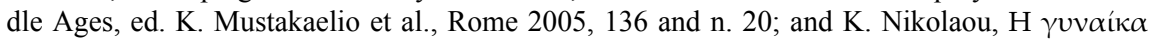

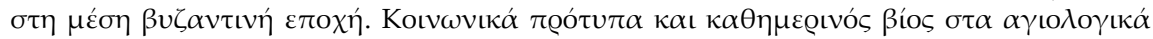


lack of education to her orphan state, and not to her infirmitas sexus. Discrimination, if discrimination it is, can be detected only in the use of the word $\dot{\alpha} v$ -

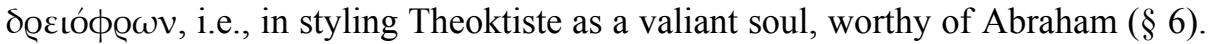
Yet, seen in context, the writer's emphasis is here not on Theoktiste's fulfilling a man's labour, but on her brave decision to sever, like Abraham, the bond of parenthood "by the sword of the spirit." ${ }^{, 26}$ In other instances, she is simply compared to the biblical and hagiographical exempla of female piety: Ruth, Anna, mother of Samuel, the mother of the Maccabees, the mother of one of the Forty Martyrs, Natalia and Priscilla. Thus, though praised for leading a life of such chastity and restraint that she virtually overcame the taint of the married state, Theoktiste is nowhere presented as having transcended the limits of her gender.

All in all, by the frequent use of scriptural quotations mostly drawn from the Old Testament, usually cited verbatim and not as allusions, Theodoros employs the straightforward form of Catechism prevailing over the sophisticated elegance of a Funeral Oration. Pronounced before his monastic community and his uncle Plato at Sakkoudion in Bithynia or, if the text dates later than $799,{ }^{27}$ at Stoudios in Constantinople this Funeral Catechism justifies its double title and rhetorical character. As a funeral speech, it was prompted by obvious sentimental reasons, without, however, reaching the dramatic heights of the letter discussed above; the introductory lamentation swiftly gives way to edification and portrayal of an idiosyncratic woman whose life both in the world and the monastery tangentially corresponded to former examples of female holiness. Addressing as he did a monastic audience, the Stoudite Father's basic endeavour was to highlight, on the one hand, disruption of the marriage and family ties, to instill, on the other hand, the idea that violent and authoritative conduct is not exclusive of, but conducive to sanctity and salvation.

As already suggested, not only in language and style, but also by retaining a view closer to mundane and earthly matters, Theodoros' Funeral Catechism had little in common with its illustrious literary precedents, namely the Orations which Gregory of Nazianzos wrote to commemorate family members (his sister Gorgonia, his brother Caesarius and his father the elder Gregory), not to mention the loftiest of all, the one celebrating the life and deeds of his close friend Basil. This text is once quoted in the Catechism but no longer used and imitated. None-

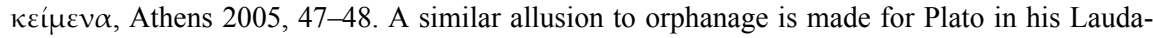
tion, but not with regard to education; see PG 99, col. 808A.

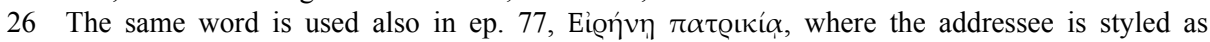

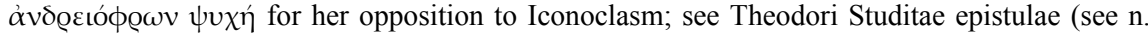
3), 190. It should be noted that in the vita $\mathrm{C}$ of Theodoros Stoudites Theoktiste is introduced as having emulated her husband and by means of her ascetical toils having made male her female nature: ed. Latyšev, in: VV 21 (1914) 250.

27 For the whole record of events see Pratsch, Theodoros Studites (see n. 15), 115-134. 
theless, like the earlier Cappadocians and the later Michael Psellos, also author of an Encomium for his Mother, Theodoros Stoudites allows us a panoramic view of mothers and families engaged in a "lofty" cause. In all aforementioned authors, this engagement becomes the basic axis upon which authors attach their underlying intention to promote the sanctification of their relatives and ultimately of themselves. We are told that Gorgonia brought her husband to her own pious way of life and that Macrina greatly contributed to prompting Basil and other members of her family to higher spiritual pursuits. ${ }^{28}$ It is this particular aspect that links up Theoktiste to the married sister of Gregory of Nazianzos and the unmarried sister of Gregory of Nyssa. Nonetheless, these women instilled moral and spiritual values in their male and female relatives, but they never operated as a driving force directing the whole family towards a total renunciation of life in the world.

In Theodoros' Catechism the mother prevails over the family and the same holds true with Psellos' mother Theodote, in whose Encomium, dated ca. 1054, she also swerves from the straight path of family life to draw herself and her husband all the more to the pursuit of ascetical practices within marriage and family, ending up to the rejection of both. Once again, there is a clear point of differentiation here: although Psellos assigns space and value to the husband and father, the latter is entirely underrated in Theodoros' account. ${ }^{29}$ We may suspect that, like Psellos' father, the husband of Theoktiste, who held a dignity in the imperial treasury $(\S 6)$, was a "secular" and practical man engaged in public affairs and not "a man of religion." 30 Although his son reserves him some encomiastic words when styling him a "Boaz by the side of Ruth" and saying that he too, deserved

28 See Or. 8, Oratio funebris ad Gorgoniam, ch. 8, ed. M. A. Calvet-Sebasti, in: Grégoire de Na-

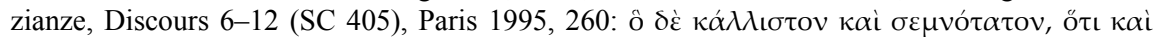

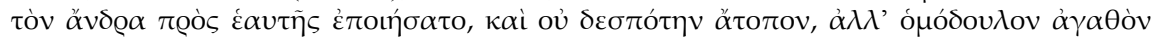

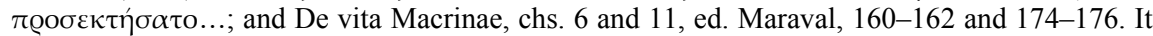
should be noted that their brothers and authors diverge on the firsthand knowledge they had about their sisters' life; whereas Gregory of Nyssa filled out the Oration to his sister Macrina with stories he had experienced or heard from others, ignorance lay behind the "rhetorical platitudes" of Gregory of Nazianzos in Gorgonia's Encomium: see R. Van Dam, Families and Friends in Late Roman Cappadocia, Philadelphia 2003, 93-96 and 109-112. For a recent analysis of the funeral oration for Gorgonia see V. Burrus, Life after Death: The Martyrdom of Gorgonia and the Birth of Female Hagiography, in: Gregory of Nazianzus: Images and Reflections, ed. J. Bjørtnes/T. Hägg, Copenhagen 2006, 153-170; and for St Gregory of Nazianzos' rhetorical viewing of his family see S. Elm, Gregory's women: Creating a philosopher's family, ibid., 171-191.

29 See J. Walker, These Things I Have Not Betrayed: Michael Psellos' Encomium of his Mother as a Defense of Rhetoric, in: Rhetorica 22 (2004) 49-101; and The Byzantine Family of Michael Psellos. Mothers and Sons, Fathers and Daughters, in: A. Kaldellis (ed.), Michael Psellos in Translation, Notre Dame 2006, forthcoming.

30 For a biographical sketch of Theodoros' father, Photeinos, see Pratsch, Theodoros Studites (see

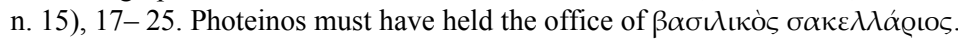


praise for leaving his wife untouched $(\S 4)$, his reluctance to go further is straightaway made visible in what comes next: religious education of daughters and sons was her own kingdom! Of him and his three brothers who are also said to have embraced the solitary life ( $(6)$ we hear no more in the narrative. ${ }^{31}$

By and large, Theodoros makes an implicit distinction between the "militant" ascetical members of the family (Plato, Theoktiste, her daughter and himself) and those who hesitantly followed them. ${ }^{32}$ Their separation, an astonishing event that made enormous impression in Constantinopolitan society, was a "voluntary separation" ( $\dot{\varepsilon} \theta \varepsilon \lambda$ ov $\tau \grave{\imath} \chi \omega \varrho \iota \sigma \mu o ́ s)$ imposed by the single will of the mother. As a term and a literary theme, separation occupies a focal position in the Catechism. Separation of death is first evoked by Theoktiste as an argument to convince her husband to live apart within marriage. More significantly, it was the heavy price for a brave and breaking action. Inserted as a personal memory and a tragic scene in the narrative, thereby somehow restraining the force of the author's rhetorical overtones, it is narrated in sentimental detail (§ 7). Just before embarking on her new life, Theoktiste ought to overcome the tears of her little son and the "tyranny" of motherhood. Later on in her life, while a nun, separation of mother from son was once again felt but in retrospect: a short meeting preceded Theodoros' and Platon's departure into exile $(\S 9) .{ }^{33}$ Finally, once again the separation of death seals a relationship that had developed in a reverse order: it transpired that the mother had become her son's spiritual daughter. In short, her escape from the world had finally subjected her to the guidance of her son, from $\alpha \pi 0 \tau \alpha \gamma \eta$ she

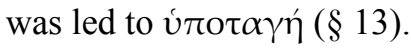

Disruption of marriage and motherhood is a significant break with the earlier "Cappadocian exempla" of female holiness and a point of divergence from Psellos' maternal praise. All mothers are highly acknowledged as having considerably contributed to the moral progress of their sons; yet, their common desire to embrace monastic life and abandon the conventional demands of marriage and family did not result in adopting similar attitudes. In Psellos, whose mother also

31 Marginalisation of the father against the mother is not an uncommon feature in vitae of the late antique and medieval period; see Pratsch, Topos (see n. 15), 68 and n. 63. This authority gained much more ground if the mother was widowed: see M. Kaplan, Hagiographie et histoire de la société, in: Les Vies des saints à Byzance. Genre littéraire ou biographie historique? Actes du colloque international philologique, Paris, 6-7-8 juin 2002, ed. P. Odorico/P. A. Agapitos (Dossiers byzantins 4), Paris 2004, 37-45.

32 Apart from the Catechism itself $(\S 6)$, this is suggested by ep. 1, addressed $\Pi \lambda \alpha \dot{\tau} \tau \omega \nu$ เ

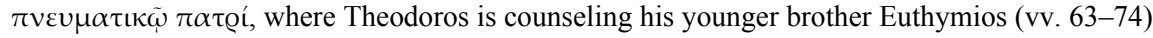
to remain steady in his monastic call; see Theodori Studitae epistulae (see n. 3), 7. On the case of Euthymios see R. Cholij, Theodore the Stoudite: the Ordering of Holiness (Oxford Theological Monographs), Oxford 2002, 17-18.

33 For the course of this exile see J.-Cl. Cheynet/B. Flusin, Du monastère Ta Kathara à Thessalonique; Théodore Stoudite sur la route de l'exil, in: REB 48 (1990) 193-211. 
ended up taking monastic vows, renunciation of marital and familial ties is regarded as an "apostasy from God". ${ }^{34}$ Exit from marriage and withdrawal to the monastic life could be enacted only following the elder daughter's death and Psellos' (rhetorical and real) coming of age. In "conformity" with Late Antique practice, mortal grief functioned as a catalyst for a departure from the world. ${ }^{35}$ Nevertheless, if the loss of his parents opened up St Anthony's - and many other holy men's - exodus to the desert, this was not so with Theoktiste's "wonderful inno-

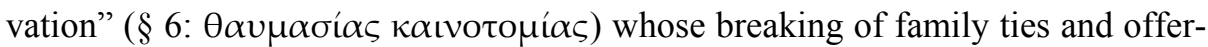
ing up her properties had no association with death. ${ }^{36}$ In his later years Theodoros expressed similar views in his letter to the protospatharia Albeneka (ep. 395), the wife of a high-ranking official related to the imperial palace. Wishing to leave her spouse for the convent, Albeneka was counseled to make him understand her cause, but, should her inner inclination prove intense enough, she was asked to follow her call in spite of his will. However, in his concluding lines the Stoudite abbot reminded her that it would have been possible to find salvation living together with a man. ${ }^{37}$

In honouring sisters and mothers, such strong personalities as the Cappadocian Fathers and Michael Psellos largely speak for themselves, emphasizing the close link between a female biography and a male autobiography, not to say "autohagiography". Though trying to disclaim implicit self-praise, giving instead the credit to his uncle Plato ( $९$ 9), Theodoros follows practically the same line: his selection of events and his account of his mother's spiritual feats is set in tandem with his own hardships and modes of persecution. ${ }^{38}$

34 See ch. 11, vv. 638-641, ed. Criscuolo (see n. 24), 107; and Walker, These Things I Have Not Betrayed (see n. 29), 98.

35 Cf. the case of Eustathios the Banker whose son's death prompted his desire for the monastic life; see S. Efthymiadis, Living in a City and Living in a Sketis: the Dream of Eustathios the Banker (BHG Nov. Auct. 1317d), in: BF 21 (1995) (= Bosphorus. Essays presented in honour of Cyril Mango), 20 and 26.

36 For parallels from later Medieval hagiography see A.-M. Talbot, The Byzantine Family and the Monastery, in: DOP 44 (1990) 119-120 (= Women and Religious Life in Byzantium [Variorum Collected Studies Series CS 733], Aldershot 2001, XIII). On Theodoros' self-presentation as a reformer of monasticism see J. Leroy, La réforme studite, in: Il monachesimo orientale. Atti del convegno di studi orientali che sul predetto tema si tiene a Roma, sotto la direzione del pontifico orientale, nei giorni 9, 10, 11 e 12 Aprile 1958 (OCA 153), Rome 1958, 184-188.

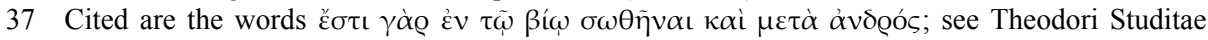
epistulae (see n. 3), 550. This letter dates from 815 to the beginning of 819 .

38 The autobiographical character of the Catechism was briefly noted by S. Efthymiadis, The Byzantine Hagiographer and his Audience in the Ninth and Tenth Centuries, in: Metaphrasis. Redactions and Audiences in Middle Byzantine Hagiography, ed. Chr. Høgel, Oslo 1996, 70; and M. Hinterberger, Autobiographische Traditionen in Byzanz (WBS XXII), Vienna 1999, 152-153. See also M. Angold, The autobiographical impulse in Byzantium, in: DOP 52 (1998) $1-17$, who notes that the first signs of autobiography in Byzantium appeared in a monastic setting, but does not treat sources earlier than the end of the $10^{\text {th }}$-beginning of the $11^{\text {th }}$ century. 
First and foremost, his laying emphasis on the violent aspects of renunciation of the worldly order ( $\dot{\alpha} \pi 0 \tau \alpha \gamma \eta \dot{)}$ ) and on "voluntary separation" was by all means also a personal experience and not an attitude privileged by his mother. In ep. 4 addressed to the abbot Nikephoros, dated to between September 795 and autumn

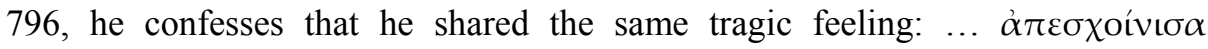

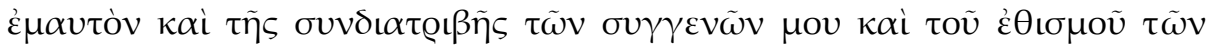

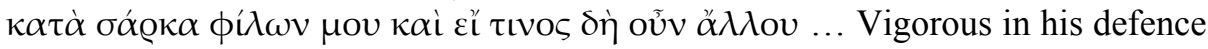
of monastic values, Theodoros developed the same ideas throughout the Catechism composed shortly afterwards. Addressing a monastic audience, he himself needed first to demonstrate that he originated from a family that instilled in him the values which he then aimed to impose on his community. His mother, invested with powers and authority hardly compatible with any woman of his age, was the guarantor of his own devotion to the monastic cause. Though it could be argued that he wrote the first eulogy of a female saint in the Byzantine Middle Ages, his concern was not so much to present a full-fledged biography as to propound a monastic ideal that was connected with and regulated secular life too. Theoktiste's acts and behaviour as a married woman and as a nun were not actually inseparable, but stood for a remarkable continuum: the way she lived in the world prefigured her days as an abbess. In this sense, the striking fact of conferring violence upon her maidservants as a lay matron anticipated her violent outbreaks in the convent. ${ }^{39}$

Women in antiquity took a leading role in society once they became mothers. For Theoktiste motherhood was the starting point for setting foot on her own path towards spiritual perfection. Monastic piety and discipline were put into action once the son came into being and common life with his mother made a beginning. It is thus no accident that her encomiast did not begin her biography with her birth, but with his own. Giving birth to Theodoros was precisely the time "when she had gained experience in the judgement of that which was good and that which was not" (§ 2). It was then that Theoktiste became conscious of her "holy identity" and of what marked her off from other women of her social class; that she rejected any sort of sorcery following the birth of a child; that she secretly abstained from meat-eating and watching theatrical performances while attending marriage feasts; that she knew one man only. Remarkably, as he would later do with the Praise of his uncle Plato, Theodoros transfers the topos of puer-senex from a "charismatic childhood" to mature age. Spiritual maturity is achieved by cutting oneself off from a society which, at least in its higher strata, "not yet out of the Dark Age," is presented as quite permissive. To be sure, this denigration of Theoktiste's social milieu can plausibly raise suspicion as ultimately resulting

39 This was first noted by Hausherr, Le moine et l' amitié (see n. 18), 339. 
from her son's endeavour to emphasize that rejecting the customary practices of her class was what estranged her from the world and directed her to a gradual spiritual advancement, first experienced in hiding (but not in negligence of her household duties) and then in actions affecting the family. Except for an allusion to her early orphaned state as a cause for her illiteracy, the author passes over in silence everything that has to do with what preceded their communal life, their $\sigma v \mu \beta i ́ \omega \sigma \iota \varsigma$, a term also used in the peroration (§ 14), again to denote the "life" expected to be shared post mortem.

Thus highlighting aspects of family life and personal emotions, Theodoros nonetheless leaves much of his mother's monastic career in the shadows, implying that it was not lacking in obstacles and vicissitudes. His account is rounded off with reference to what constituted a reversing of the mother-and-son relationship. Betraying his propensity for coining new words, ${ }^{40}$ Theodoros styled his

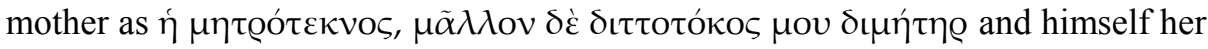
lord and father, thereby denoting what he viewed as a reversal of the natural order and his own second birth. As hagiographers did in many Lives of saints, Theodoros assigned to the mother a crucial role in paving her son's path of holiness, a prize that Theoktiste had first won for herself. Pronouncing this particular kind of hagiographic praise was a prolegomenon to establishing a holy lineage that was to be perpetuated by those engaged in the harsh monastic life: a sort of prefiguration of the holy portrait of himself.

40 On Theodoros' art as a wordsmith see G. Fatouros, Zur Sprache des Theodoros Stoudites, in: Lexicographica Byzantina. Beiträge zum Symposion zur byzantinischen Lexikographie (Wien, 1.-4. 3. 1989), ed. W. Hörandner/E. Trapp (BV 20), Vienna 1991, 123-128. 


\section{Text}

E cod. Parisino graeco 1491 (= P), ff. 94-103

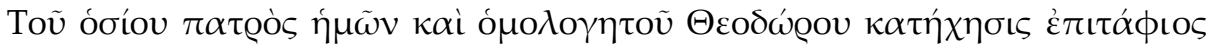

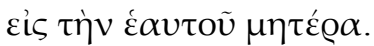

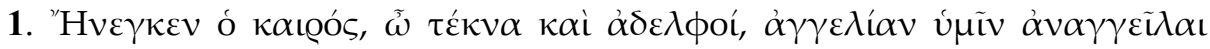

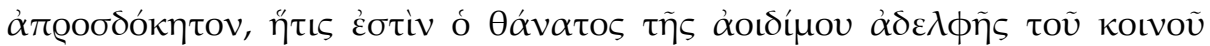

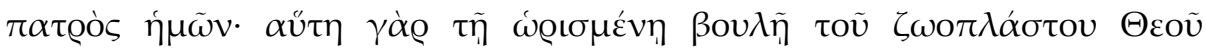

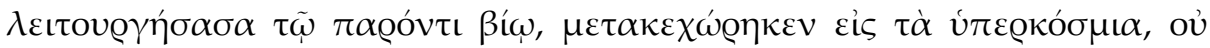

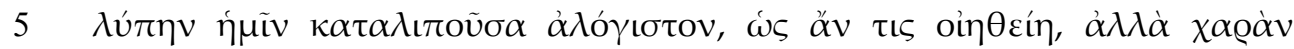

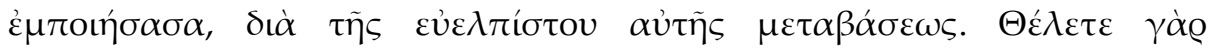

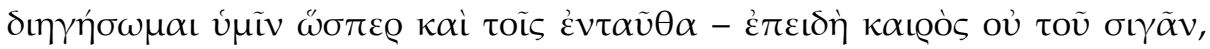

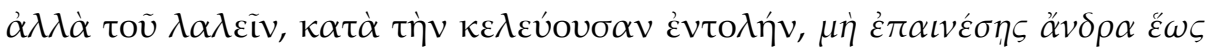

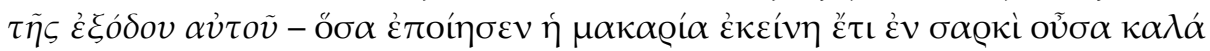

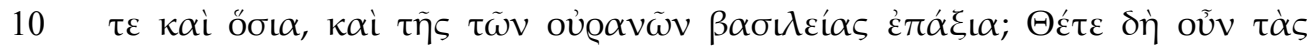

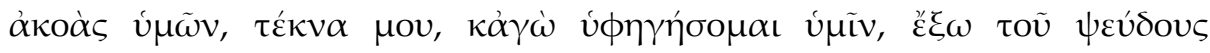

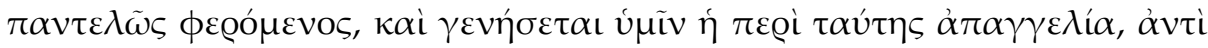

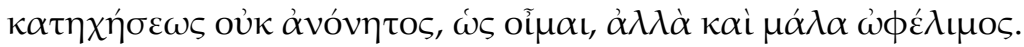

7-8 cf. Eccles. 3,7 8-9 Sir. 27,7

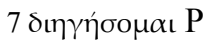

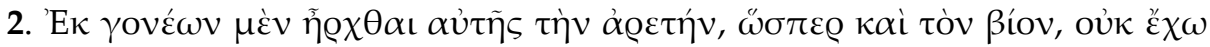

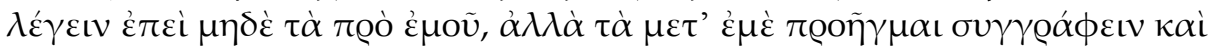

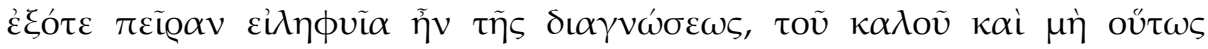

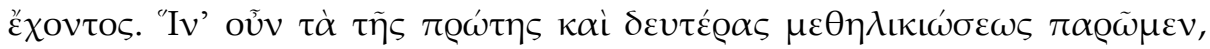

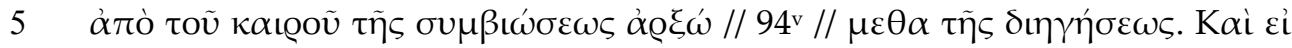

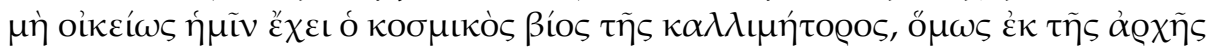

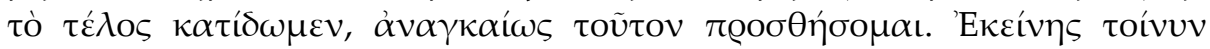

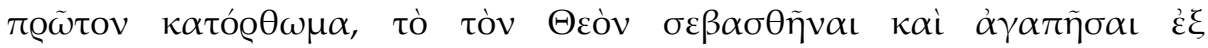

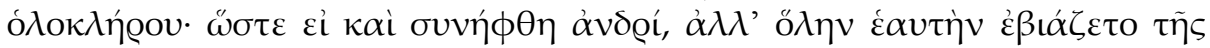

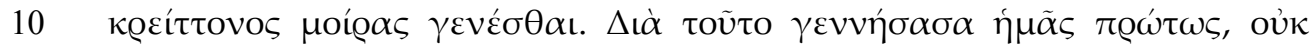

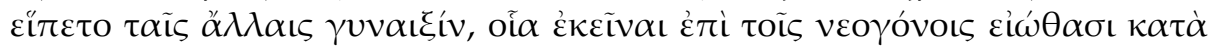

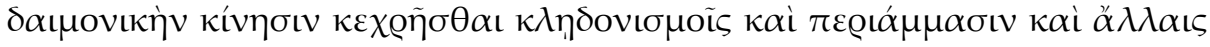

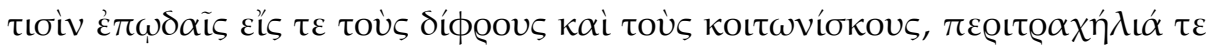

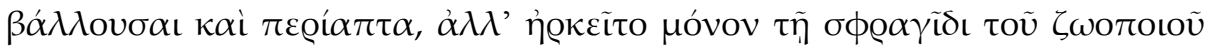

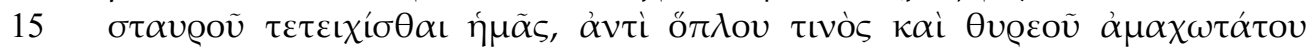

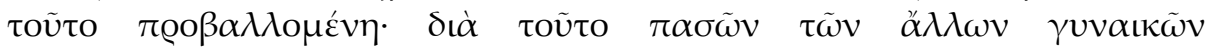




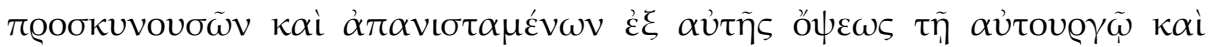

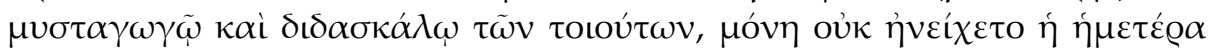

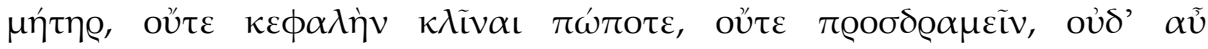

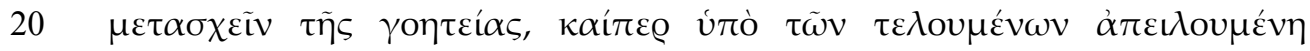

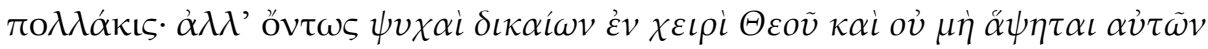

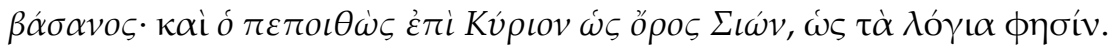

8-9 cf. Deut. 6.5 12-14 cf. Io. Chrysostomi, Ad illuminandos catechesis 2, PG 49, 231; In epistulam ad Ephesios, PG 62,48; In epistulam ad Thessalonicenses PG 62, 41215 cf. Ps. 34.2. 21-22 Sap. 3,1 22 Ps.124 (125),1

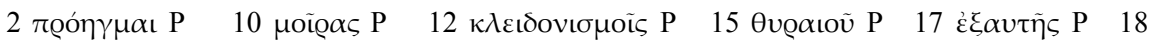

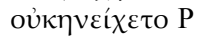

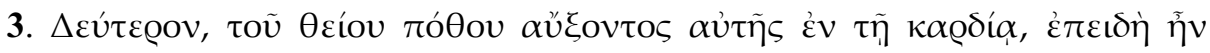

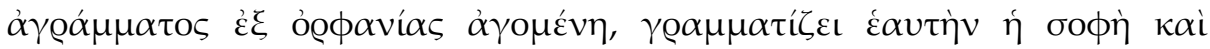

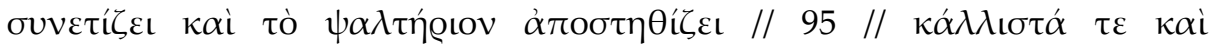

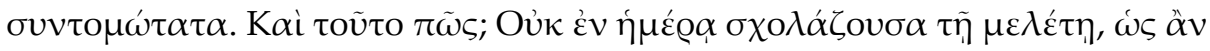

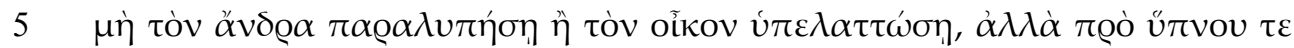

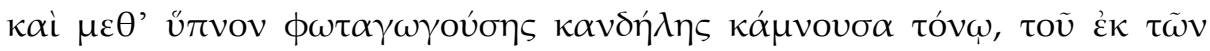

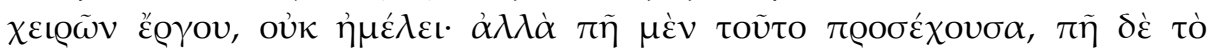

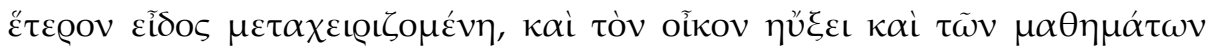

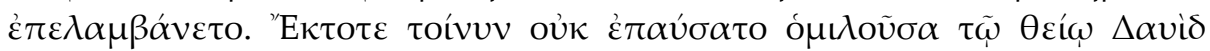

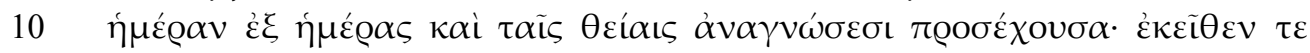

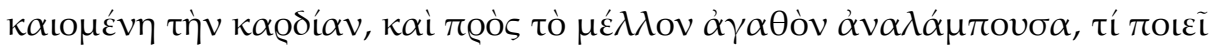

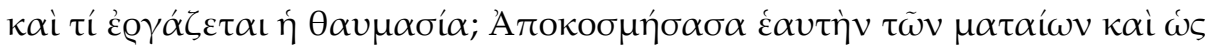

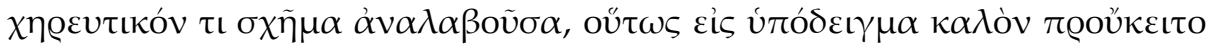

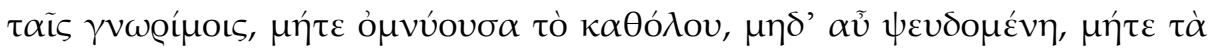

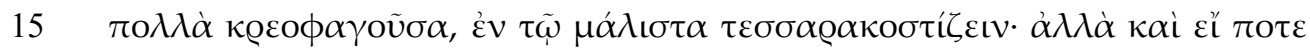

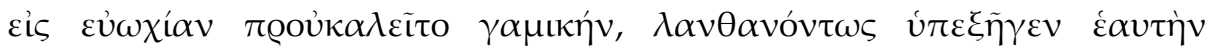

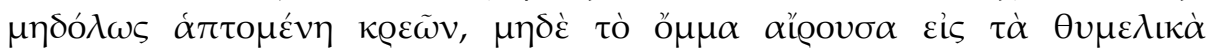

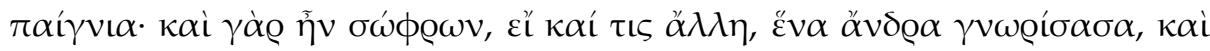

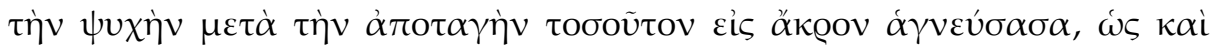

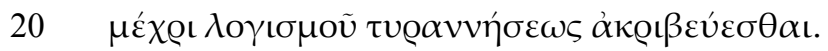

10 cf. Ps. 60,911 cf. Lk. 24,32

$14 \mu \eta \dot{\delta} \delta \mathrm{P} \quad 17 \mu \eta \dot{\gamma} \delta \dot{\mathrm{P}}$ 


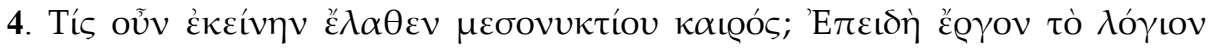

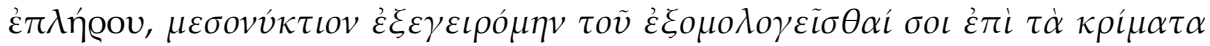

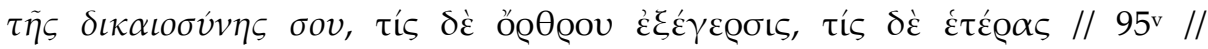

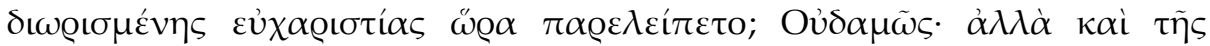

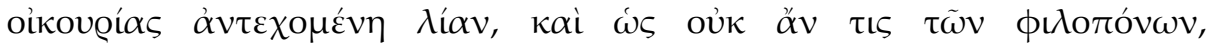

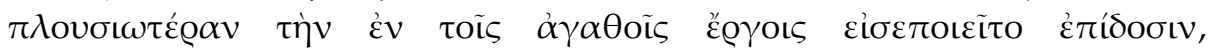

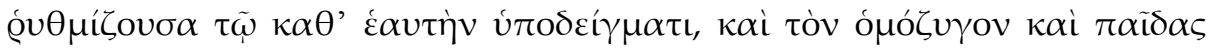

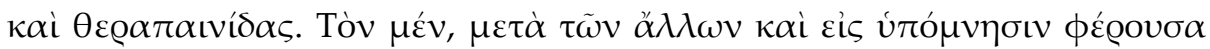

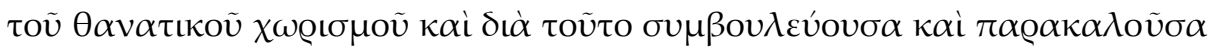

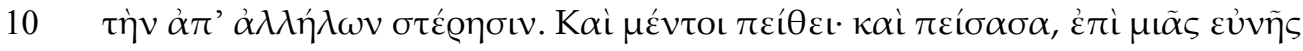

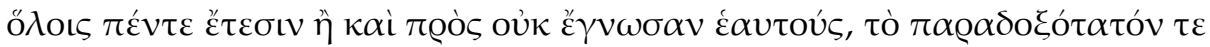

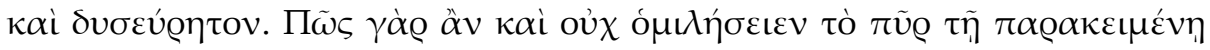

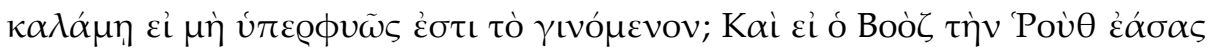

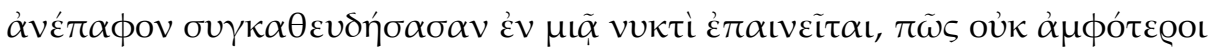

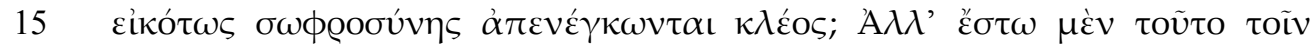

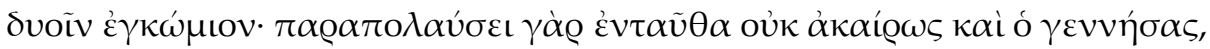

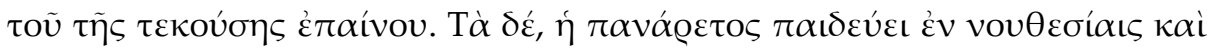

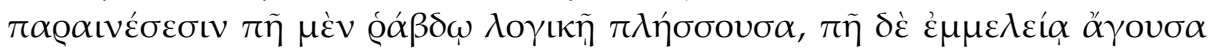

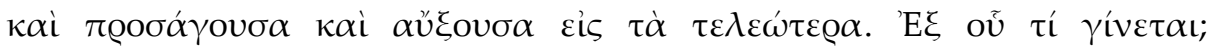

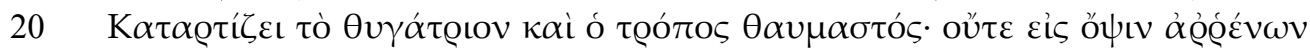

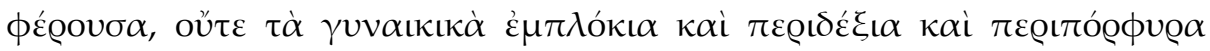

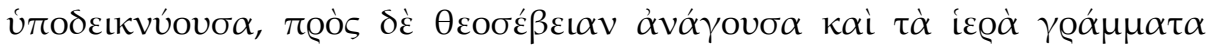

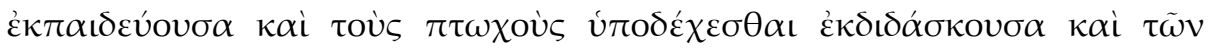

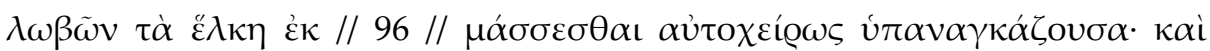

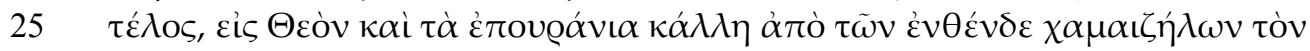

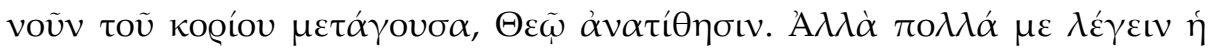

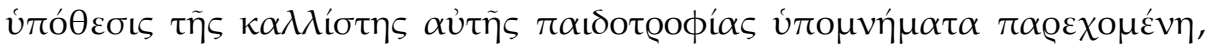

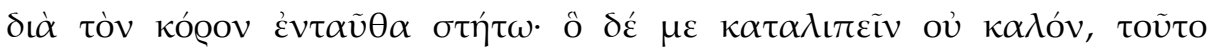

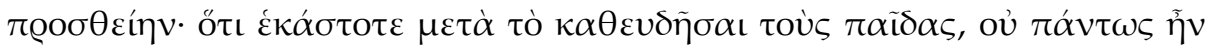

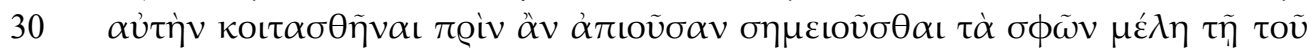

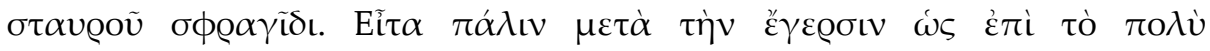

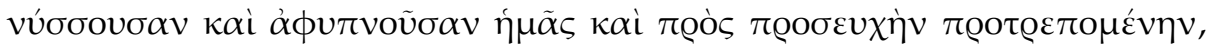

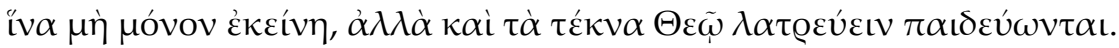

\section{2-3 Ps. 118 (119),62 13-14 cf. Ruth 3,7-14}

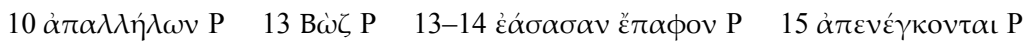

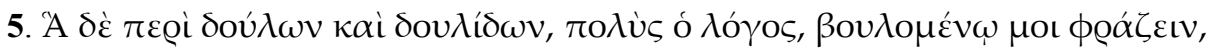

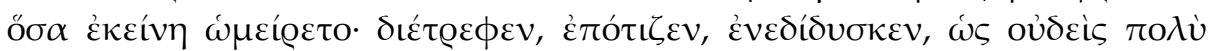




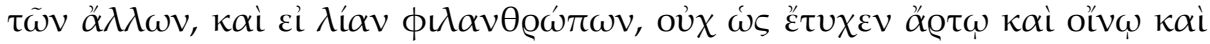

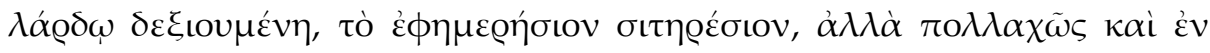

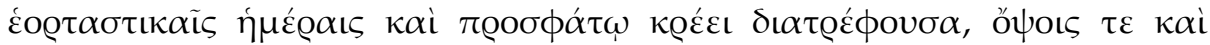

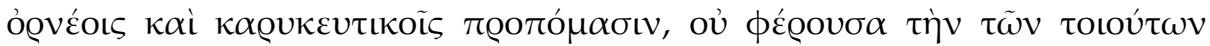

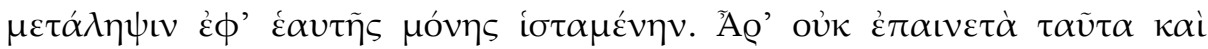

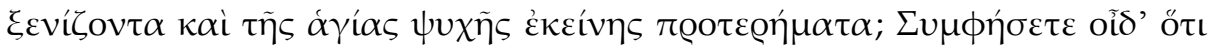

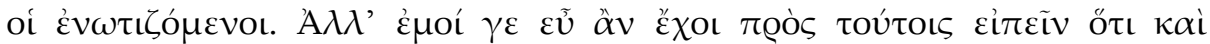

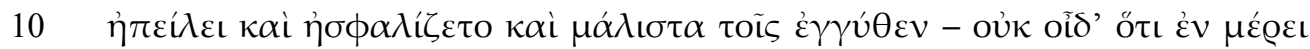

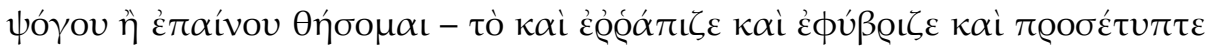

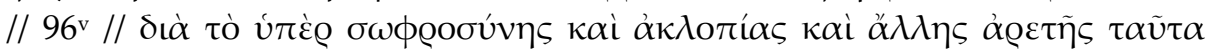

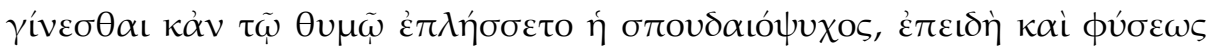

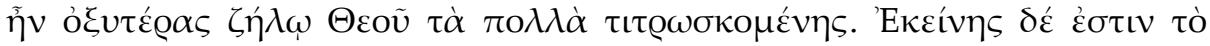

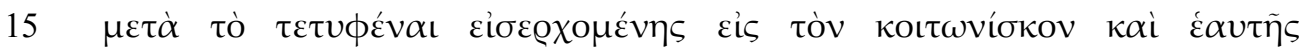

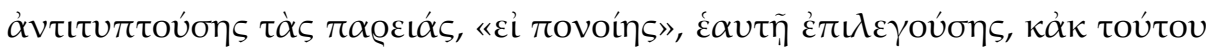

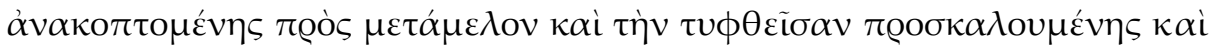

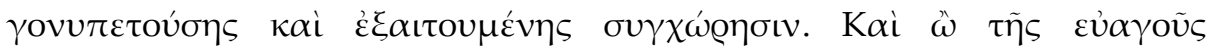

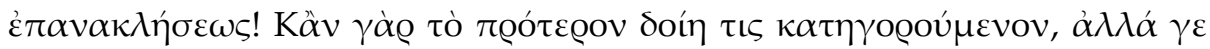

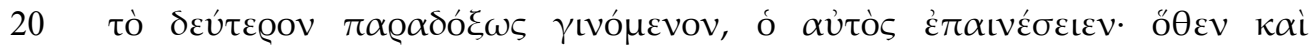

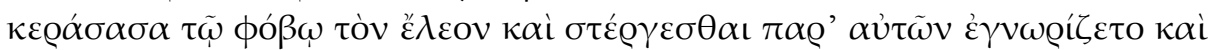

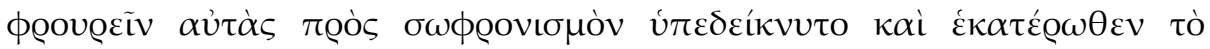

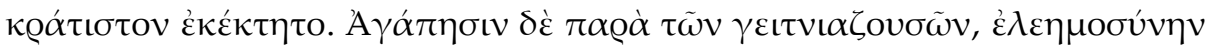

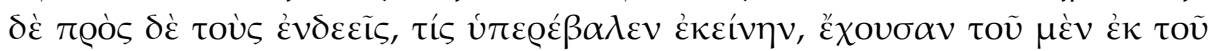

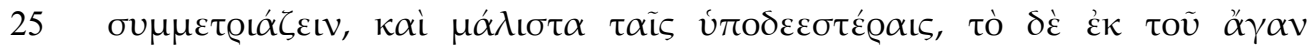

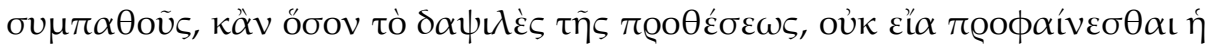

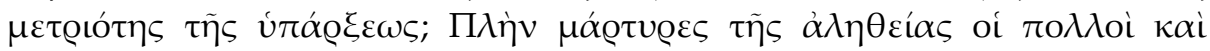

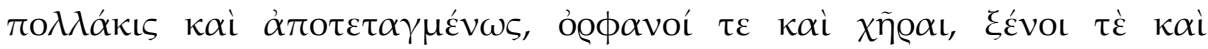

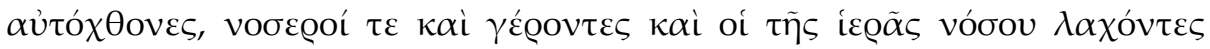

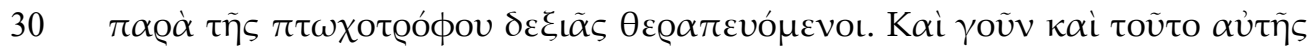

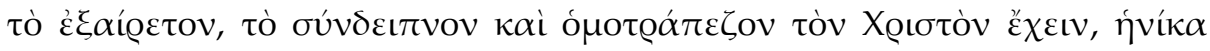

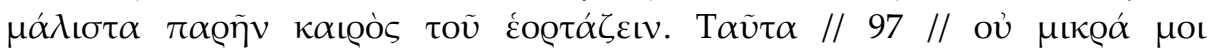

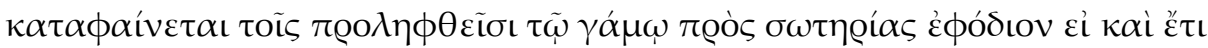

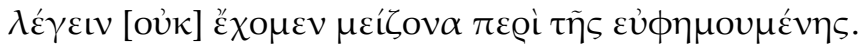

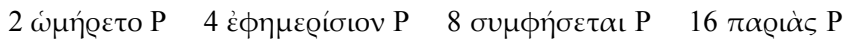

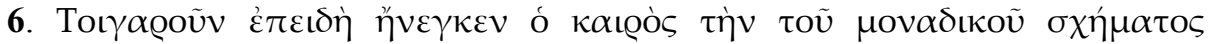

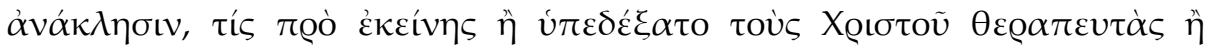

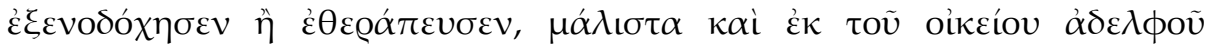

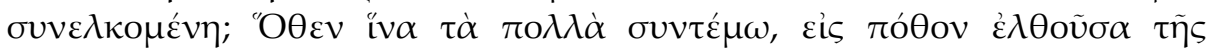




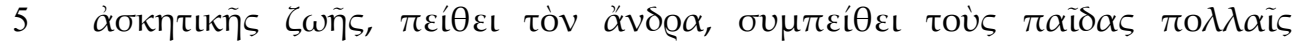

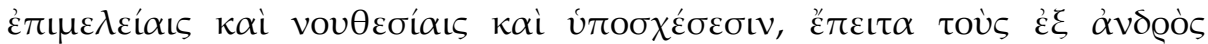

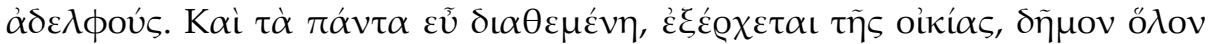

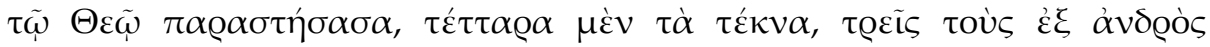

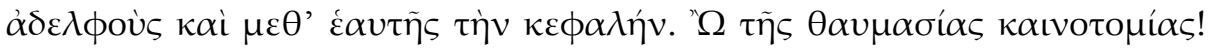

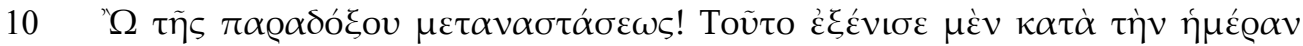

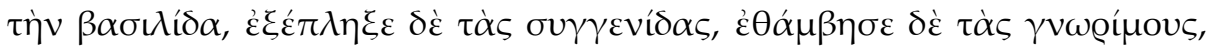

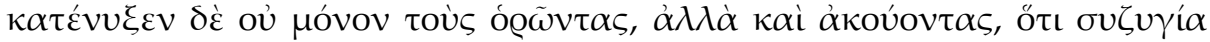

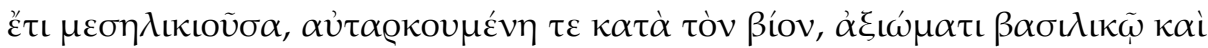

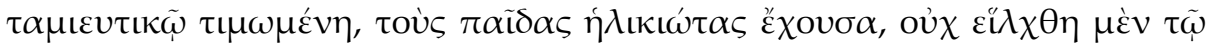

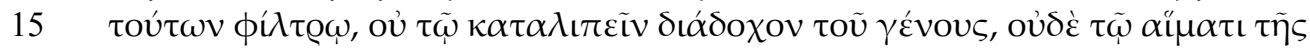

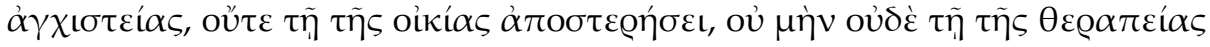

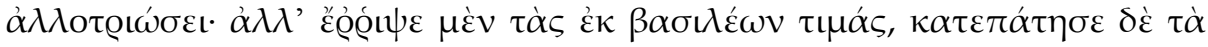

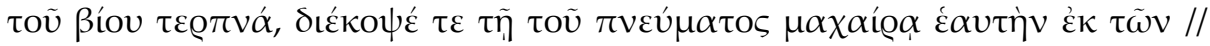

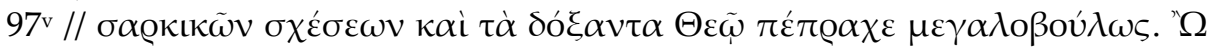

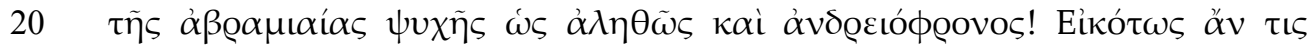

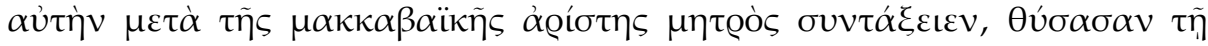

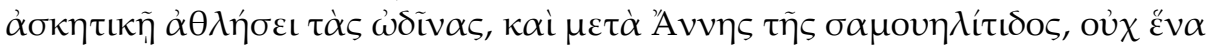

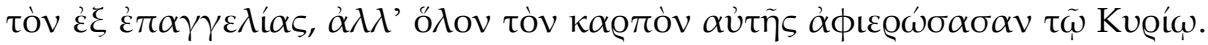

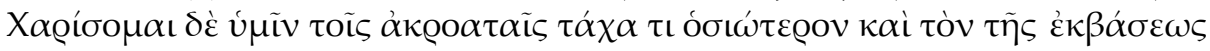

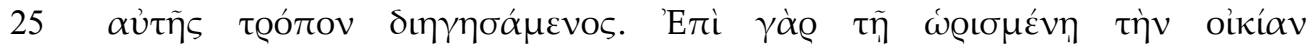

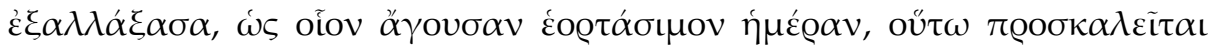

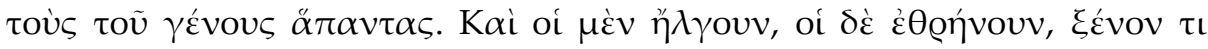

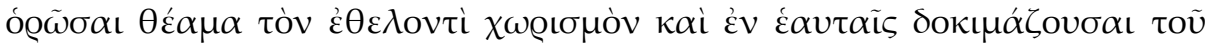

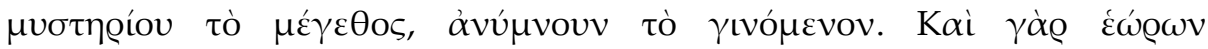

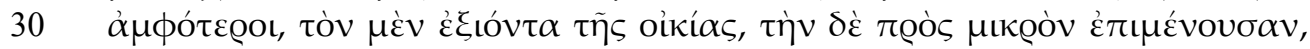

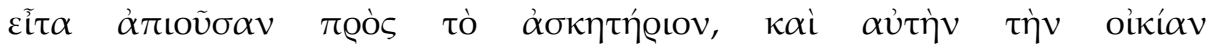

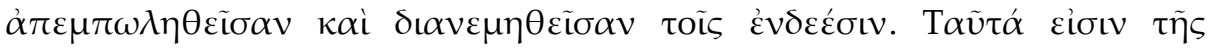

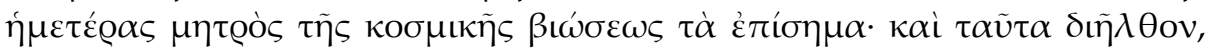

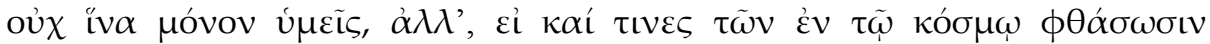

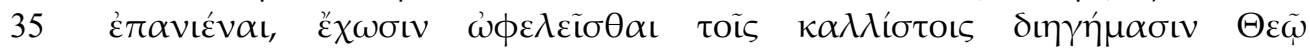

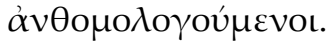

18 cf. Mat. 10, 34sqq. et Eph. 6,17 20 cf. Gen 22,10sqq. $\quad 21-22$ cf. II Macc. 7,20-23 2223 cf. I Reg. $1,11$.

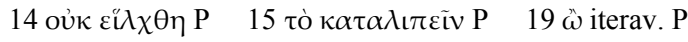




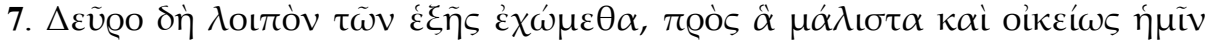

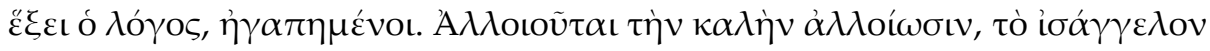

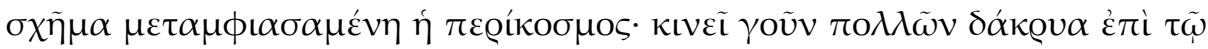

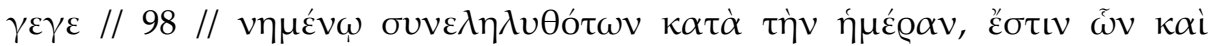

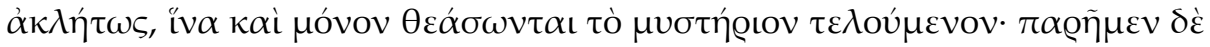

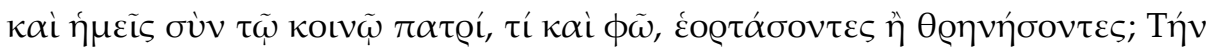

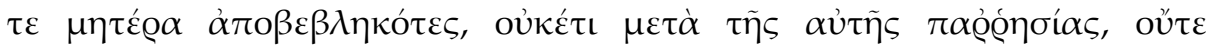

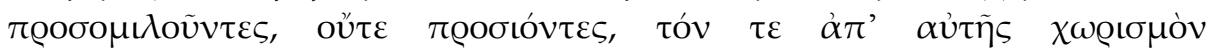

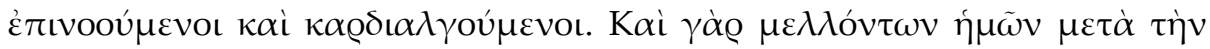

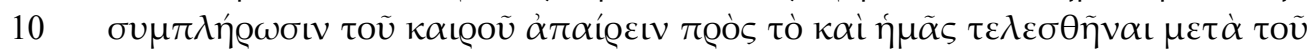

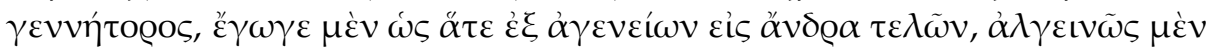

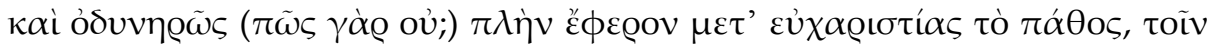

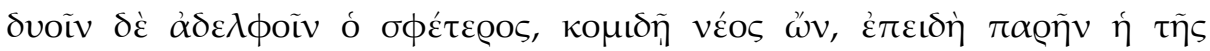

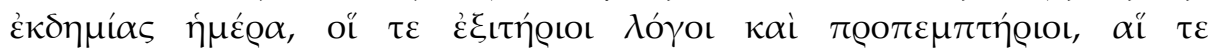

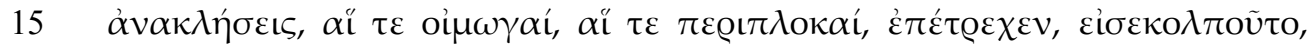

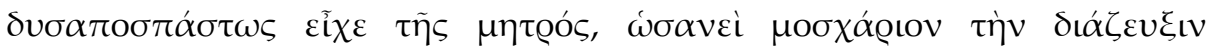

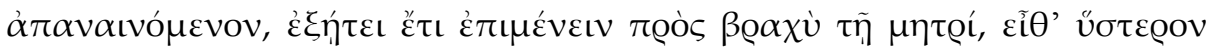

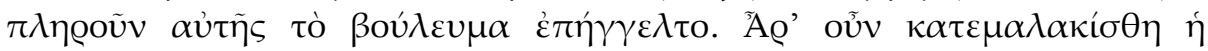

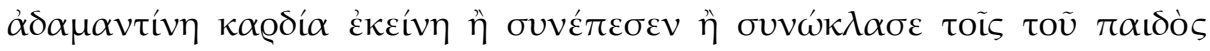

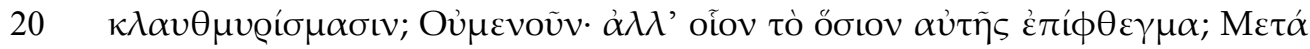

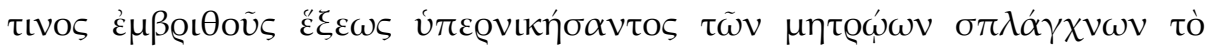

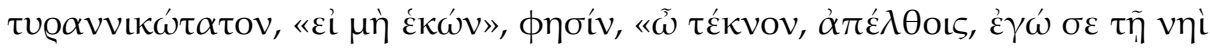

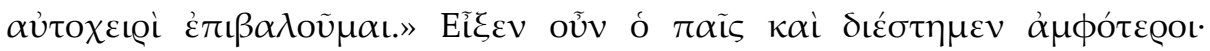

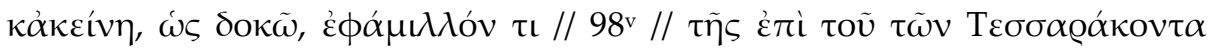

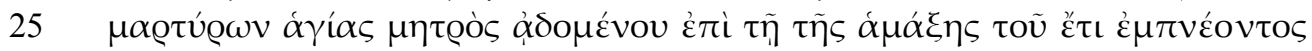

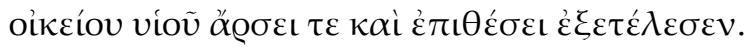

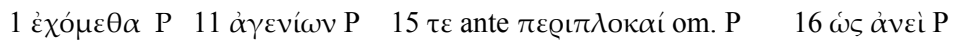




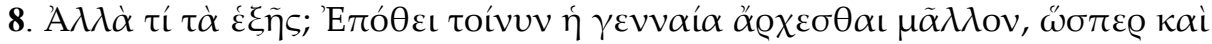

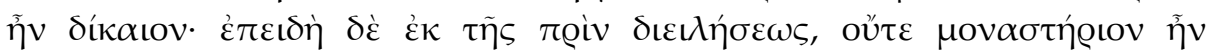

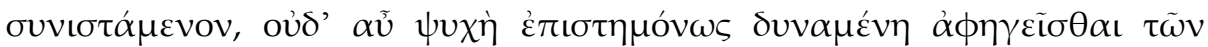

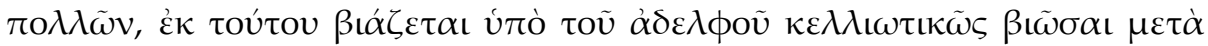

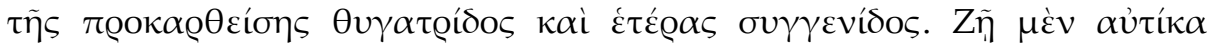

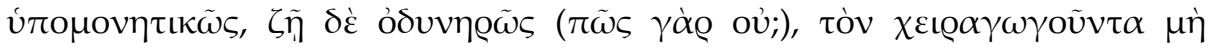

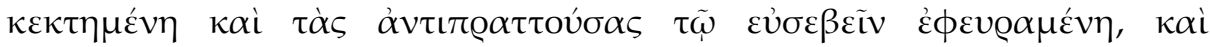

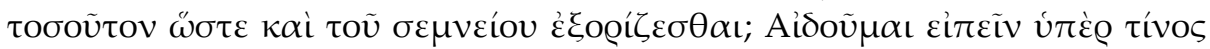

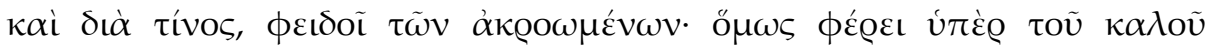

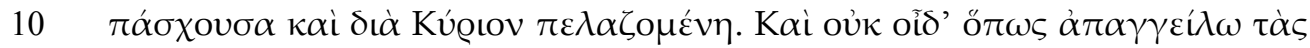

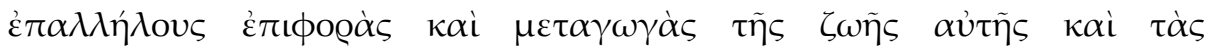

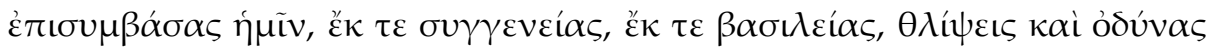

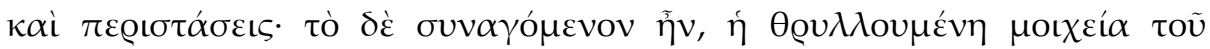

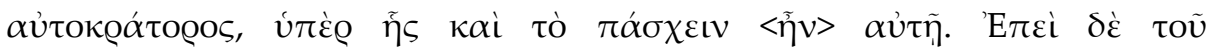

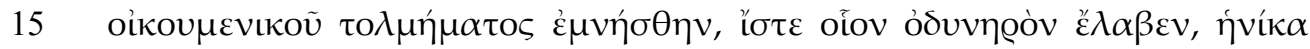

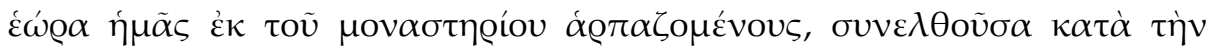

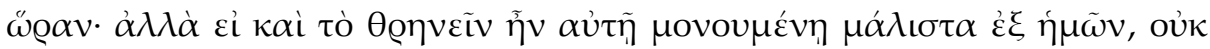

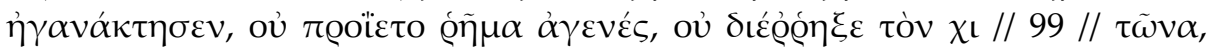

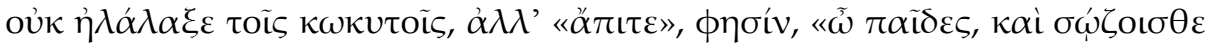

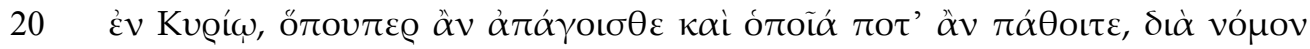

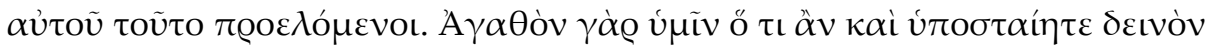

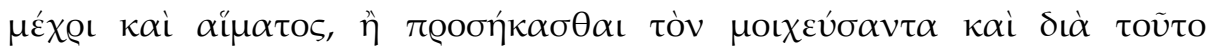

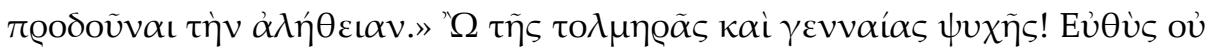

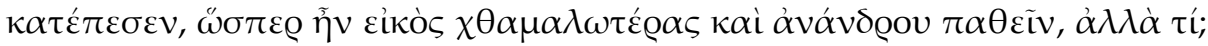

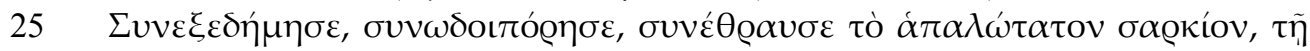

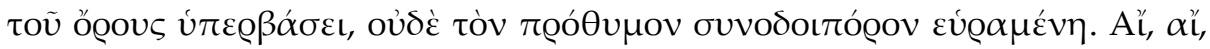

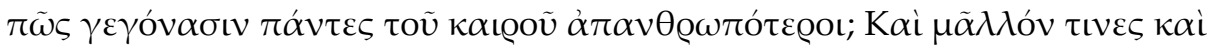

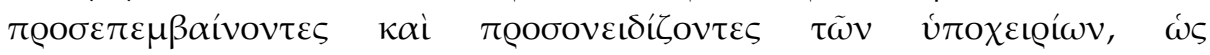

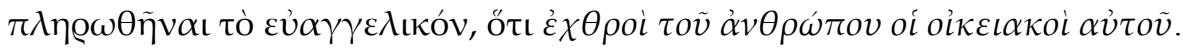

29 Mat. 10,36

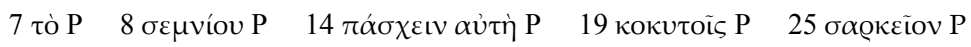




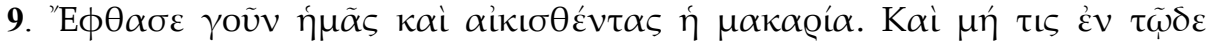

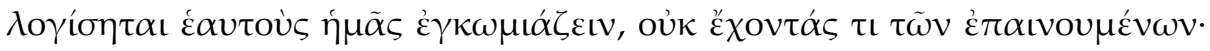

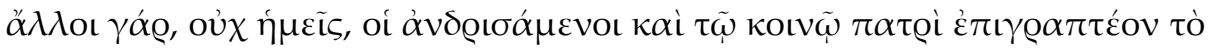

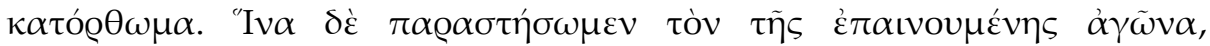

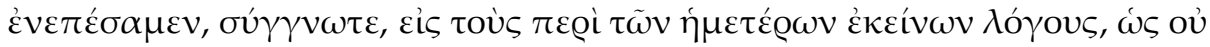

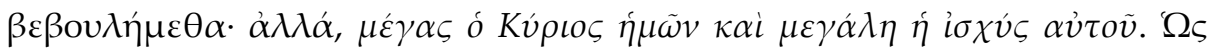

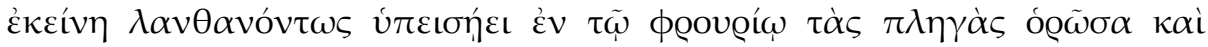

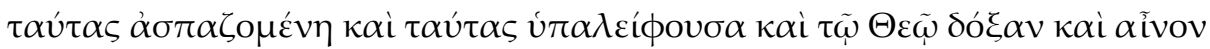

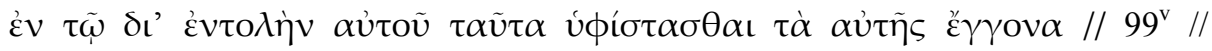

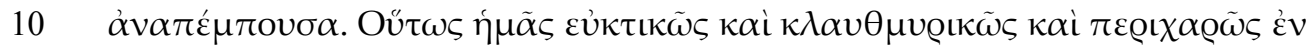

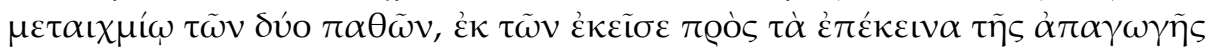

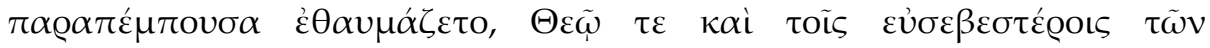

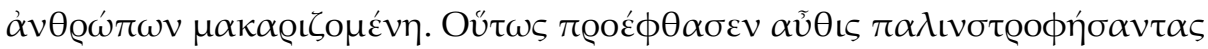

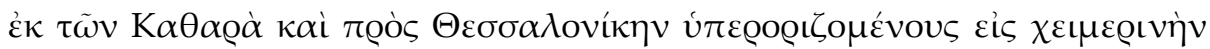

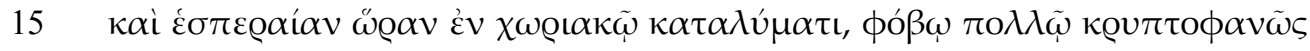

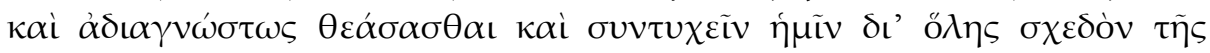

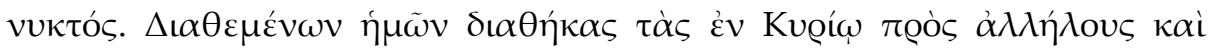

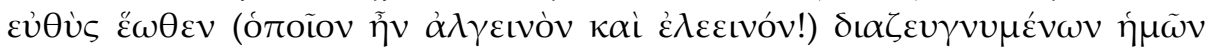

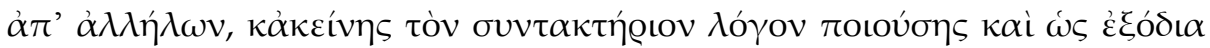

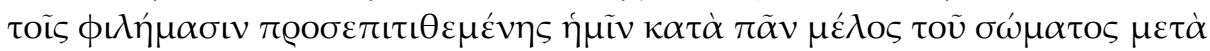

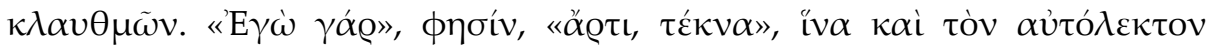

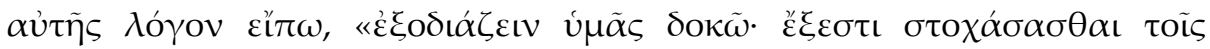

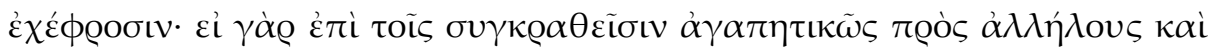

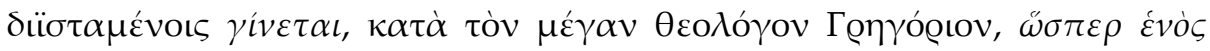

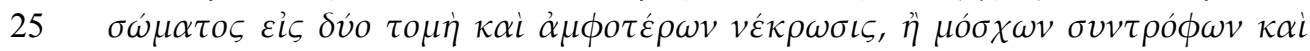

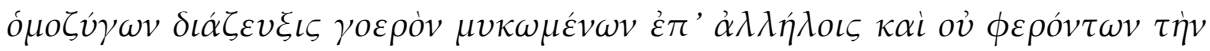

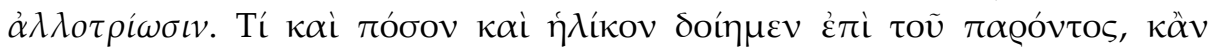

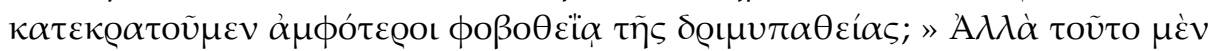

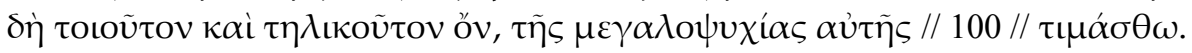

3 cf. 1 Cor. 16,13 6 Ps. 146 (147), 5 24-27 Greg. Nazianzeni, Funebris oratio in laudem Basilii magni, no 43, 24,4.3, ed. J. Bernardi, Grégoire de Nazianze, Discours 42-43 (SC 384), Paris 1992, 180.

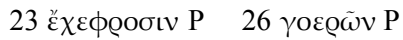

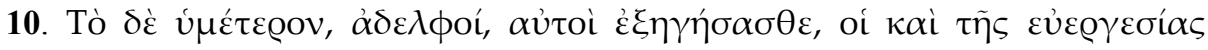

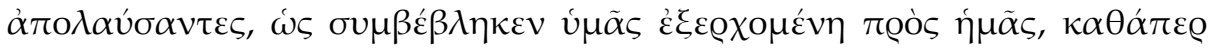

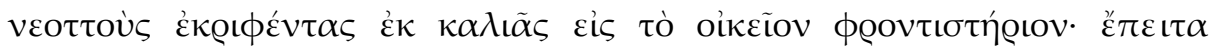




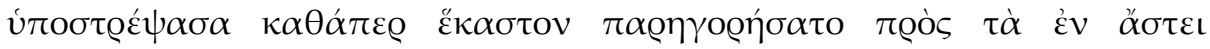

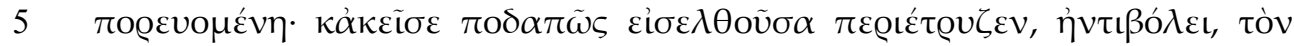

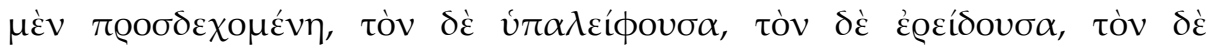

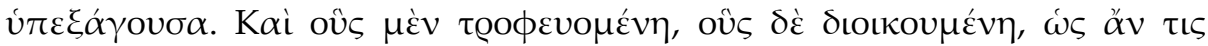

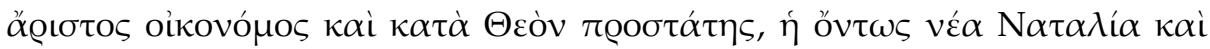

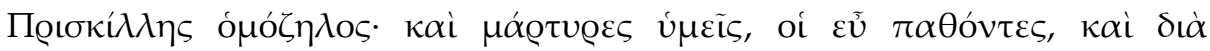

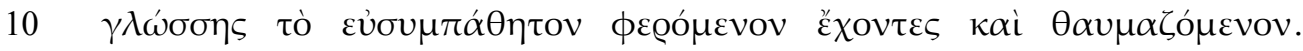

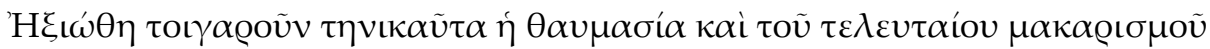

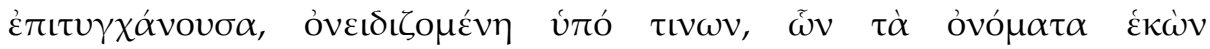

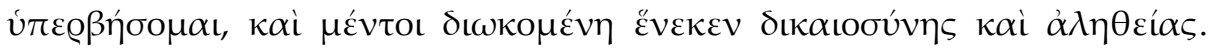

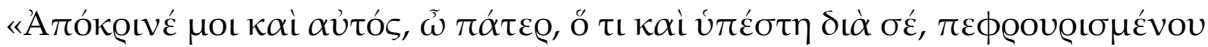

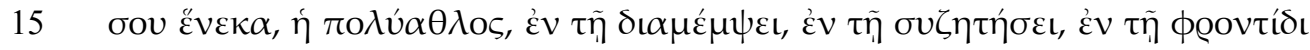

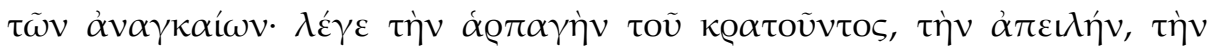

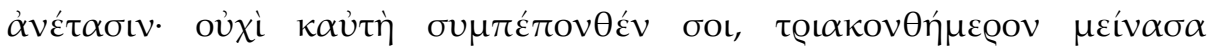

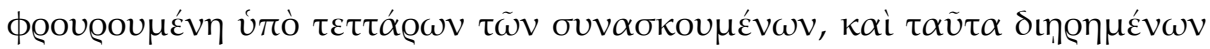

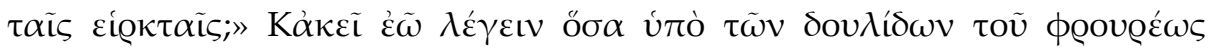

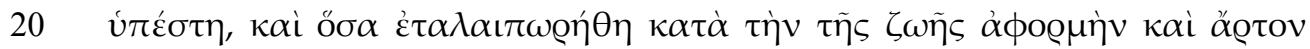

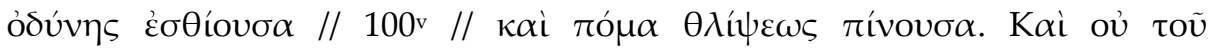

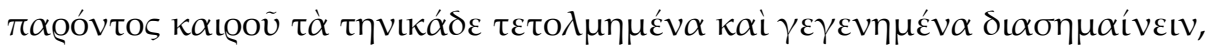

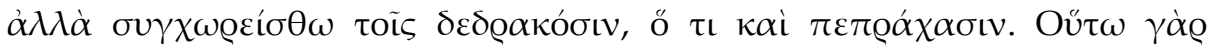

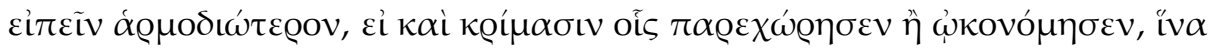

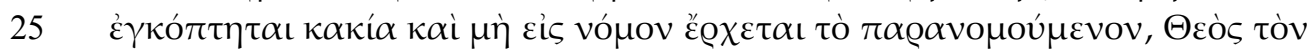

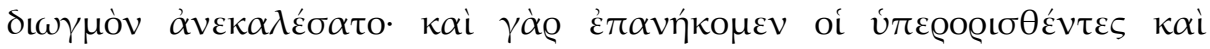

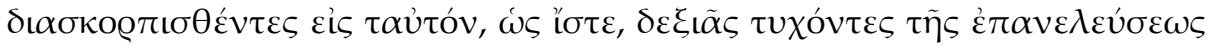

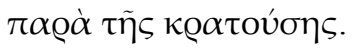

9 cf. Rom. 16,3 13 cf. Mat. 5,10 20-21 cf. Ps. 126,2 et Ps. 101,10 25 cf. Io. Chrysostomi, In Genesim, PG 53, 186.

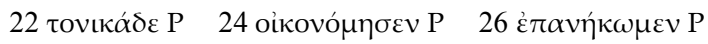

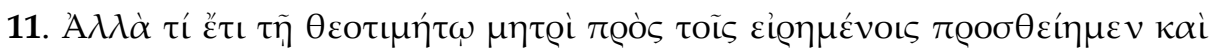

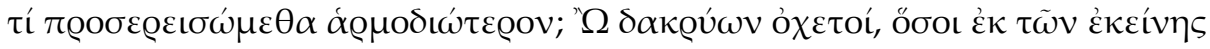

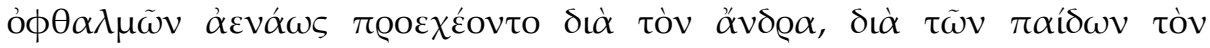

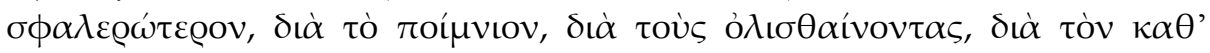

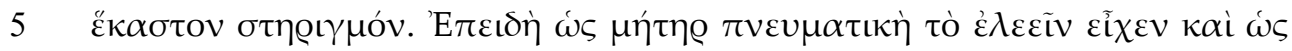

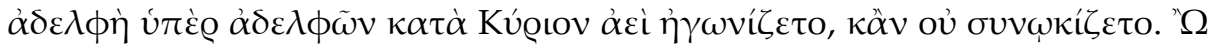

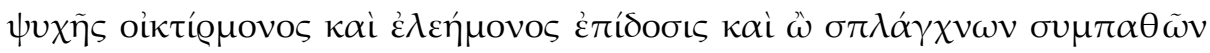

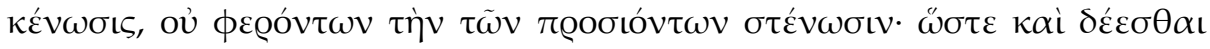

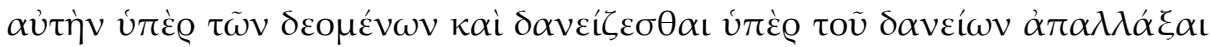




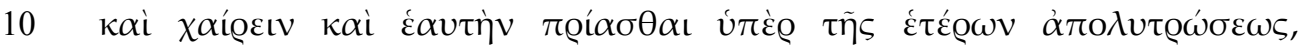

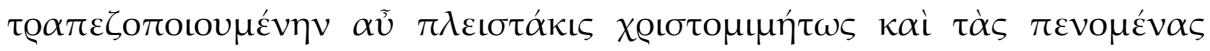

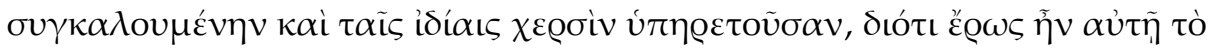

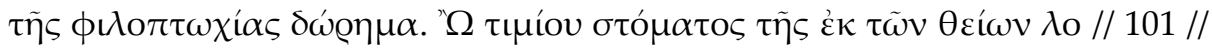

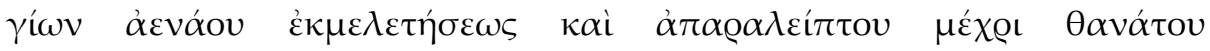

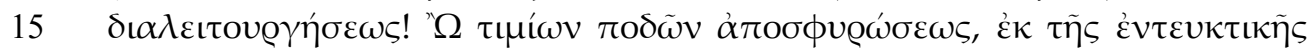

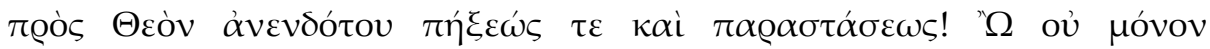

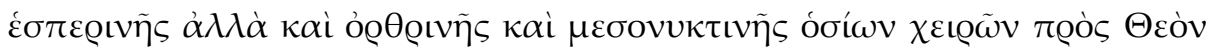

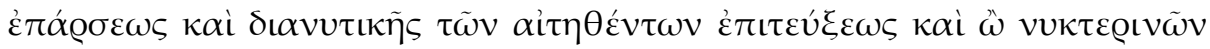

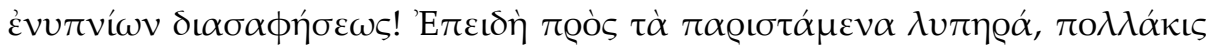

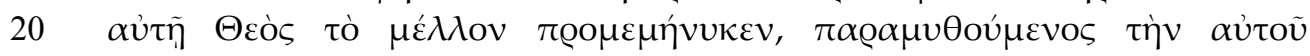

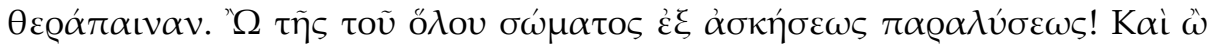

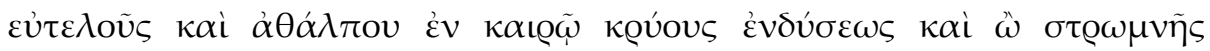

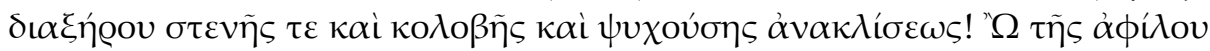

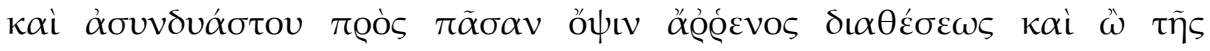

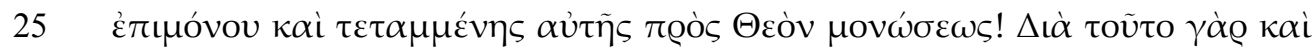

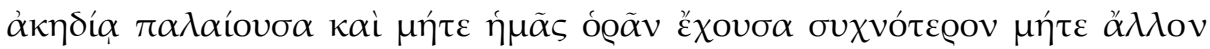

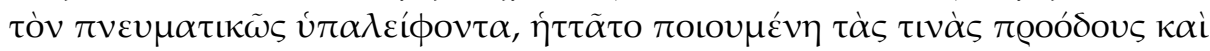

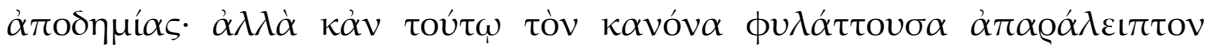

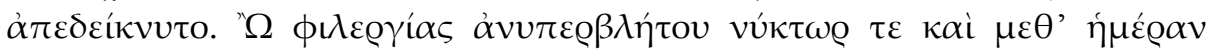

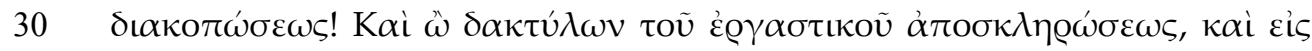

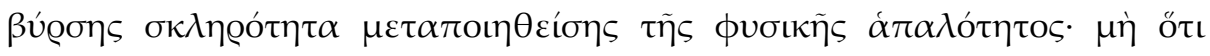

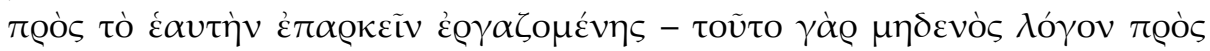

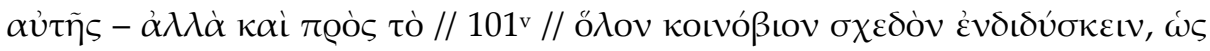

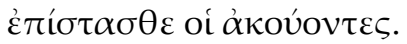

\section{4-5 cf. 2 Petr. 3,17 17-18 cf. Ps. 140,2}

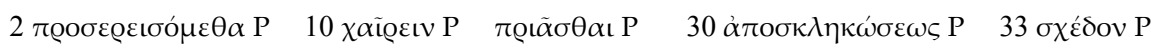

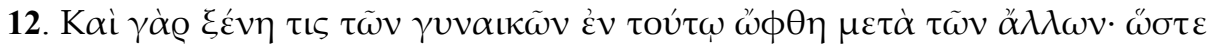

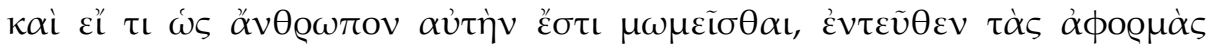

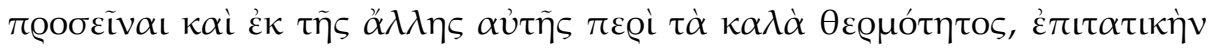

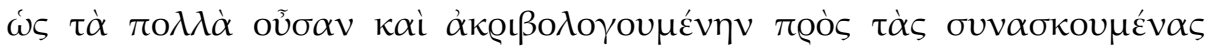

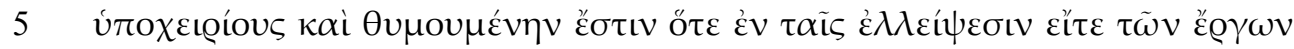

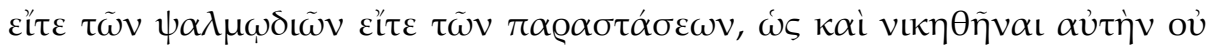

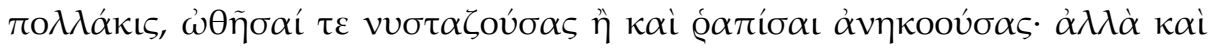

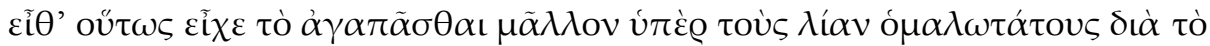

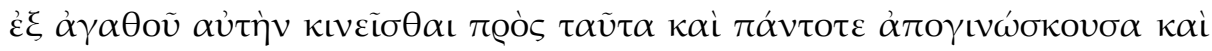

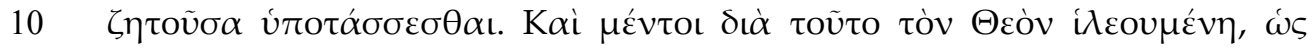




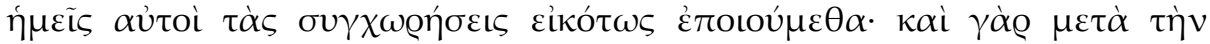

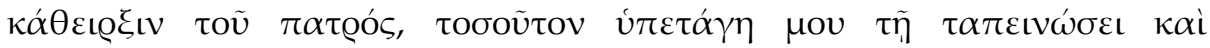

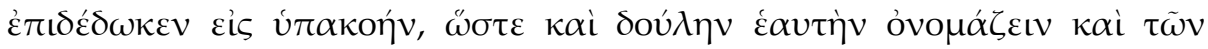

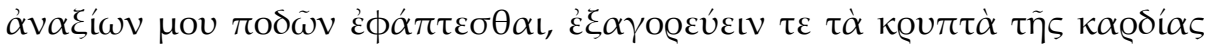

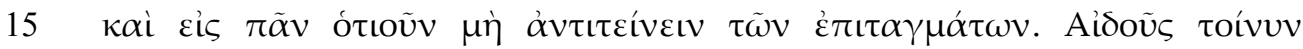

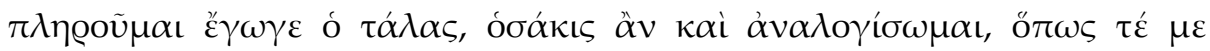

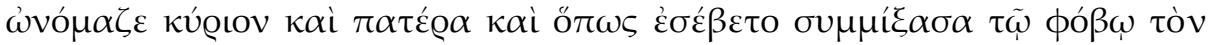

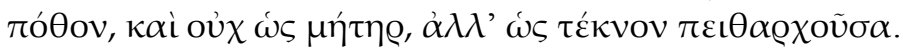

14 cf. Ps. 43,22

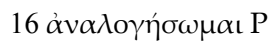

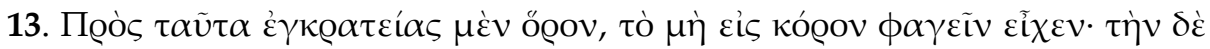

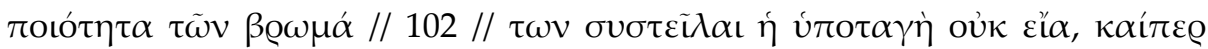

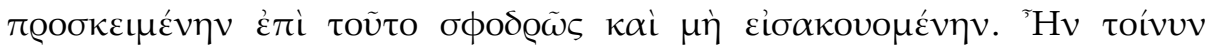

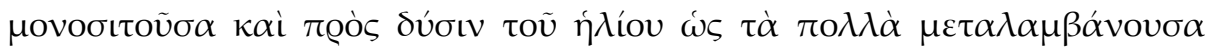

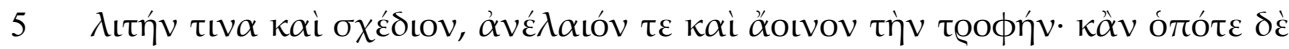

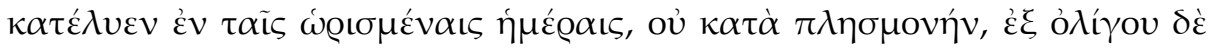

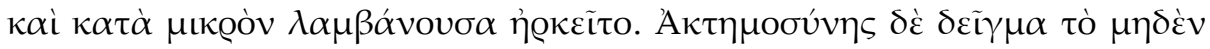

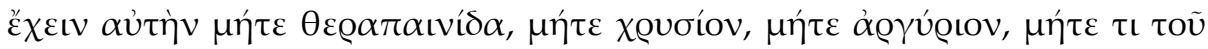

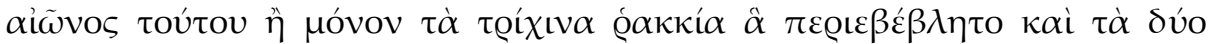

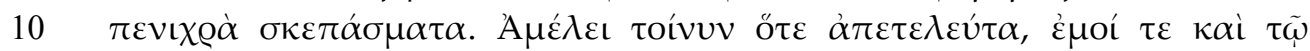

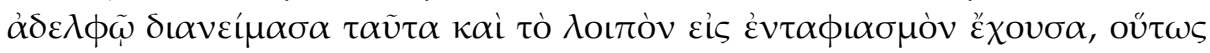

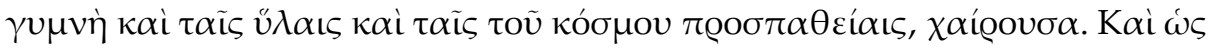

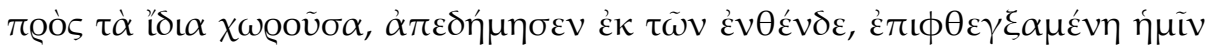

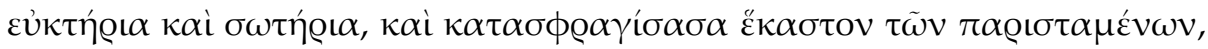

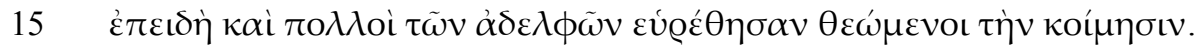

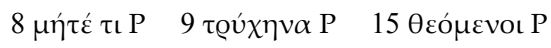

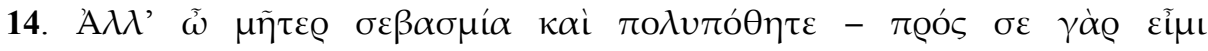

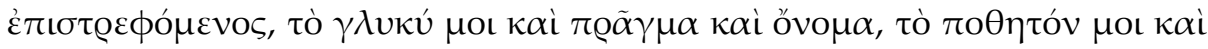

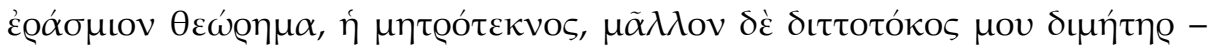

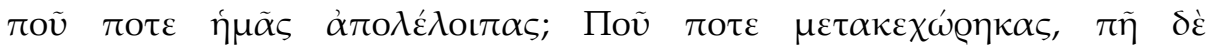

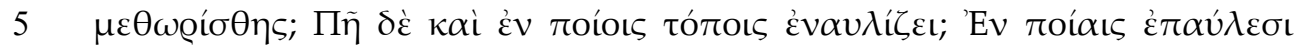

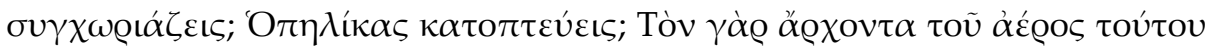

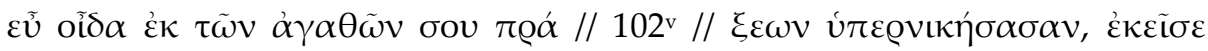

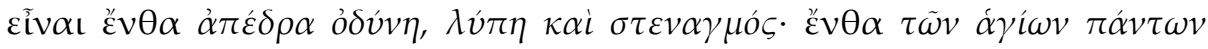

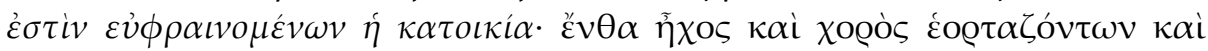




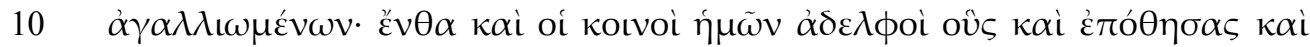

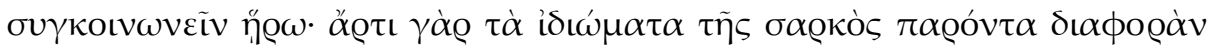

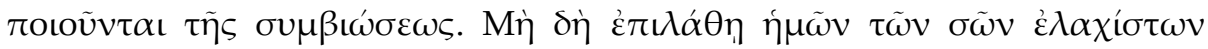

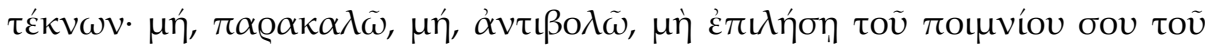

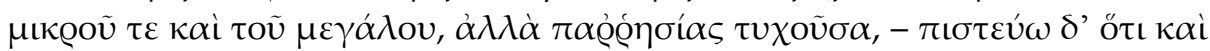

$15 \varepsilon \dot{\pi} \pi \iota \varepsilon \tau u ́ \chi \eta \kappa \alpha \varsigma-\sigma \tau \tilde{\eta} \theta \mathrm{\iota} \kappa \alpha i$ ì

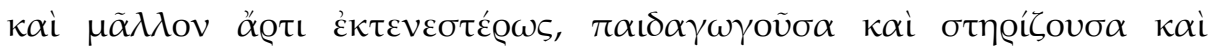

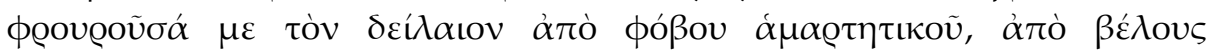

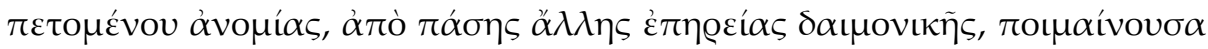

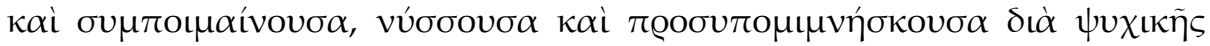

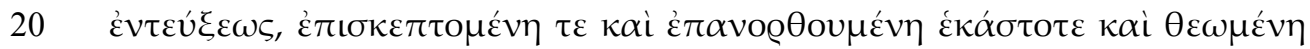

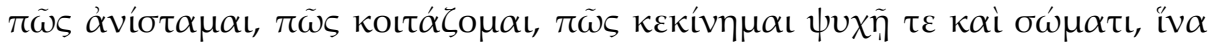

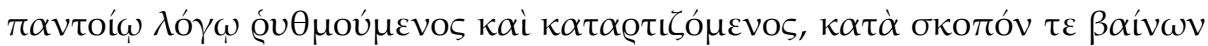

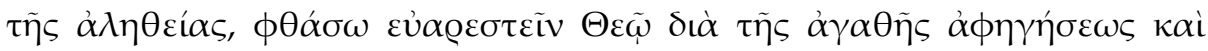

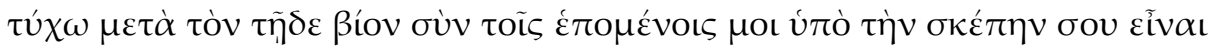

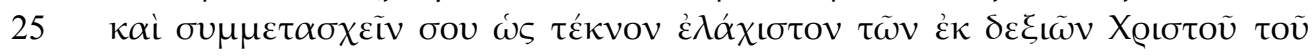

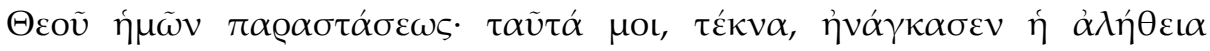

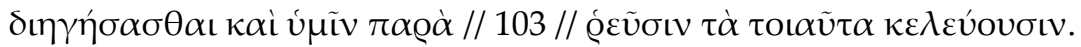

6 cf. Eph. 2,2 8 Is. 51,11; cf. kontakion officii funebris $\quad 8-9$ Ps. $86,7 \quad 9-10$ cf. Ps. $41,5 \quad 17-$ 18 cf. Ps. 90,5

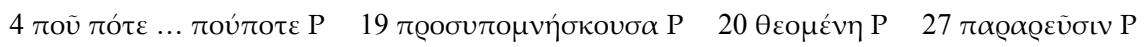


Index verborum

\begin{tabular}{|c|c|c|c|}
\hline 'Avv $\alpha$ & 6,22 & $\dot{\alpha} \pi \alpha v i ́ \sigma \tau \alpha \mu \alpha \iota$ & 2,17 \\
\hline Boó & 4,13 & $\dot{\alpha} \pi \alpha \varrho \alpha ́ \alpha \varepsilon ı \pi \tau \circ \varsigma$ & $\mathbf{1 1}, 14,28$ \\
\hline 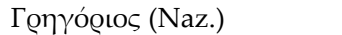 & $\mathbf{9}, 24$ & 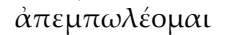 & 6,32 \\
\hline 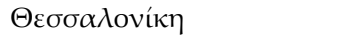 & $\mathbf{9 , 1 4}$ & $\dot{\alpha} \pi \mathbf{\gamma} \gamma \iota \omega \omega ́ \sigma \kappa \omega$ & 12,9 \\
\hline K $\alpha \theta \alpha \varrho \alpha ́$ & 9,14 & $\dot{\alpha} \pi о \delta \eta \mu i ́ \alpha$ & $\mathbf{1 1}, 28$ \\
\hline 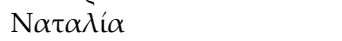 & $\mathbf{1 0}, 8$ & $\dot{\alpha} \pi$ тобоб $\mu \dot{\varepsilon} \omega$ & 3,12 \\
\hline 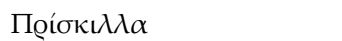 & 10,9 & 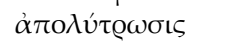 & 11,10 \\
\hline Poú $\theta$ & 4,13 & $\dot{\alpha} \pi 0 \sigma \kappa \lambda \eta ́ \emptyset \omega \sigma \iota \varsigma$ & 11,30 \\
\hline$\sum \mathrm{t} \omega \dot{v} v$ & 2,22 & $\alpha \dot{\pi} \pi \sigma \tau \varepsilon \dot{0})_{\sigma \iota \varsigma}$ & 6,16 \\
\hline \multirow[t]{2}{*}{ 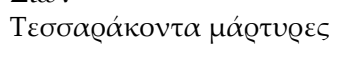 } & 7,24 & $\alpha \dot{\pi} \pi о \tau \tau \eta \theta i ́ \zeta \omega$ & 3,3 \\
\hline & & 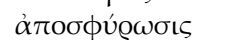 & 11,15 \\
\hline$\dot{\alpha} \beta \mathrm{\varrho} \alpha \mu \iota \alpha \tilde{\imath} о \varsigma$ & 6,20 & $\dot{\alpha} \pi \mathrm{o} \alpha \gamma \eta \dot{n}$ & 3,19 \\
\hline 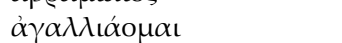 & 14,10 & $\dot{\alpha} \pi 0 \tau \varepsilon \lambda \varepsilon v \tau \alpha ́ \alpha$ & 13,10 \\
\hline$\dot{\alpha} \gamma \alpha ́ \pi \eta \sigma \iota \varsigma$ & 5,23 & $\dot{\alpha} \pi 0 \tau \varepsilon \tau \alpha \gamma \mu \varepsilon ́ v \omega \varsigma$ & 5,28 \\
\hline 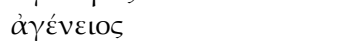 & 7,11 & $\alpha \dot{\pi} \pi 0 \phi \varepsilon ́ \varrho O \mu \alpha \mathrm{\iota}$ & 4,15 \\
\hline$\alpha \gamma \gamma \varepsilon v ́ \omega$ & 3,19 & ă $0 \sigma ı \varsigma \varsigma$ & 7,26 \\
\hline$\dot{\alpha \gamma \varrho \alpha ́ \alpha \mu \alpha \tau о \varsigma}$ & 3,2 & ăotv & 10,4 \\
\hline 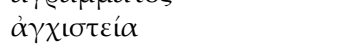 & 6,16 & 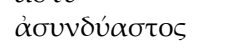 & 11,24 \\
\hline 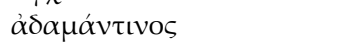 & 7,19 & 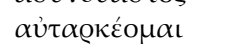 & 6,13 \\
\hline$\dot{\alpha} \delta \iota \gamma \gamma \nu \omega \sigma \tau \omega \varsigma$ & 9,16 & $\alpha$ & 8,14 \\
\hline 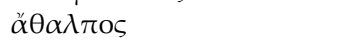 & 11,22 & $\alpha u ̉ \tau o ́ \lambda \varepsilon \kappa \tau о \zeta$ & 9,21 \\
\hline$\alpha \grave{i} \hat{\prime} \zeta \mathrm{o} \mu \alpha \iota$ & 9,1 & 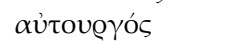 & 2,17 \\
\hline$\dot{\alpha} \kappa \eta \delta i ́ \alpha$ & 11,26 & 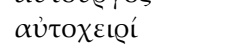 & 7,23 \\
\hline$\dot{\alpha} \kappa \lambda \eta \dot{\eta} \tau \omega \varsigma$ & 7,4 & 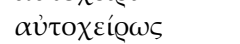 & 4,24 \\
\hline$\alpha \dot{\alpha} \lambda$ o $\pi \dot{\imath} \alpha$ & $\mathbf{5}, 12$ & $\alpha \dot{\tau} \tau o ́ \chi \theta \omega \nu$ & 5,29 \\
\hline$\dot{\alpha} \kappa \varrho \iota \beta \varepsilon v ́ \omega$ & 3,20 & $\alpha \phi \eta ́ \gamma \eta \sigma \iota \varsigma$ & 14,23 \\
\hline 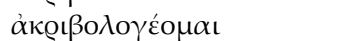 & 12,4 & 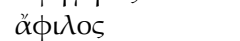 & 11,23 \\
\hline 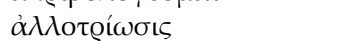 & 6,$17 ; 9,27$ & 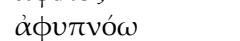 & 4,32 \\
\hline 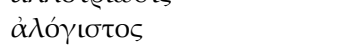 & 1,5 & & \\
\hline 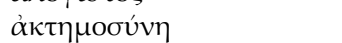 & 13,7 & $\beta \alpha \sigma u \lambda i ́ \varsigma$ & 6,11 \\
\hline$\alpha \mu \alpha \varrho \tau \eta \tau \iota \kappa o ́ s$ & 14,17 & ßúgơ & 11,31 \\
\hline 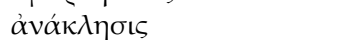 & 6,$2 ; 7,15$ & & \\
\hline 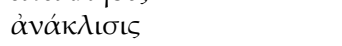 & 11,23 & $\gamma \alpha \mu t \kappa o ́ \varsigma$ & 3,16 \\
\hline$\dot{\alpha} v \alpha \kappa o ́ \pi \tau о \mu \alpha \iota$ & $\mathbf{5}, 17$ & $\gamma \varepsilon v \nu \eta ́ \tau \omega \mathrm{Q}$ & 7,10 \\
\hline$\dot{\alpha} v \alpha \pi \varepsilon \dot{\varepsilon} \mu \pi \omega$ & 9,10 & $\gamma о \eta \tau \varepsilon i ́ \alpha$ & 2,20 \\
\hline$\dot{\alpha} v \delta \varrho \varepsilon เ o ́ \phi \varrho \omega \nu$ & 6,20 & & $\mathbf{5}, 18$ \\
\hline$\alpha v \delta \varrho i \zeta o \mu \alpha \iota$ & 9,3 & $\gamma \varrho \alpha \mu \mu \alpha \tau i \zeta \omega$ & 3,2 \\
\hline 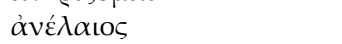 & 13,5 & 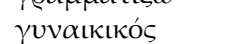 & 4,21 \\
\hline 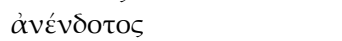 & 11,16 & & \\
\hline$\dot{\alpha} v \varepsilon ́ \tau \alpha \sigma \iota \varsigma$ & 10,17 & $\delta \varepsilon \xi \xi$ เóo $\mu \alpha \mathrm{\iota}$ & 5,4 \\
\hline$\dot{\alpha} v \eta \kappa о \varepsilon \varepsilon \omega$ & 12,7 & $\delta \mathrm{\iota} \alpha \zeta \varepsilon v ́ \gamma v v \mu \alpha \iota$ & 9,18 \\
\hline 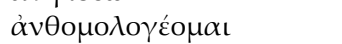 & 6,36 & 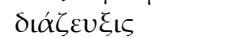 & 9,26 \\
\hline 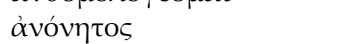 & 1,13 & $\delta \iota \alpha \kappa o ́ \pi \omega \sigma \iota \varsigma$ & 11,30 \\
\hline$\dot{\alpha} v \tau \iota \beta \mathrm{o} \lambda \varepsilon \dot{\varepsilon} \omega$ & 10,$5 ; 14,13$ & $\delta \iota \alpha \lambda \varepsilon ı \tau o u ́ g \gamma \eta \sigma \iota \varsigma$ & 11,15 \\
\hline$\dot{\alpha} v \tau \iota \pi \varrho \alpha ́ \tau \tau \tau \omega$ & 8,7 & $\delta เ \alpha ́ \mu \varepsilon \mu \psi \iota \varsigma$ & 10,15 \\
\hline$\dot{\alpha} v \tau \iota \tau u ́ \pi \tau \omega$ & 5,16 & 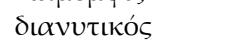 & 11,18 \\
\hline$\dot{\alpha} v v \pi \varepsilon ́ \mathrm{Q} \beta \lambda \eta \tau о \varsigma$ & 11,29 & 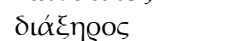 & 11,23 \\
\hline ăotvos & 13,5 & $\delta ı \alpha \sigma \alpha ́ \phi \eta \sigma \iota \varsigma$ & 11,19 \\
\hline$\dot{\alpha} \pi \alpha v \alpha i ́ v o u \alpha \iota$ & 7,17 & $\delta\llcorner\alpha \sigma \eta \mu \alpha i ́ v \omega$ & 10,22 \\
\hline
\end{tabular}


$\delta ı \delta \alpha \sigma \kappa \alpha \lambda \mathrm{o \varsigma}$

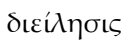

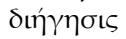

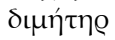

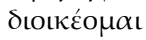

ঠıттото́коร

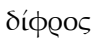

$\delta \varrho \iota \nu \pi \alpha \dot{\theta} \theta \varepsilon\llcorner\alpha$

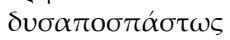

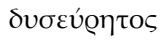

ह้ $\gamma$ rovov

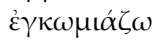

$\varepsilon \theta \varepsilon \lambda \mathrm{ov} \tau \dot{1}$

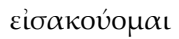

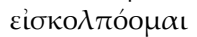

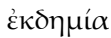

$\dot{\varepsilon} \kappa \delta \iota \delta \alpha ́ \alpha \kappa \omega$

$\dot{\varepsilon} \kappa \mu \alpha ́ \alpha \sigma \sigma \mathrm{\alpha} \alpha \mathrm{t}$

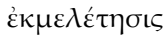

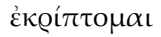

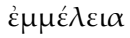

$\dot{\varepsilon} \mu \pi i ́ \pi \tau \omega$

$\dot{\varepsilon} \mu \pi \lambda$ óкเоV

$\varepsilon \dot{\varepsilon} v \alpha v \lambda i ́ \zeta \omega$

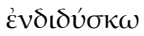

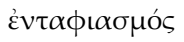

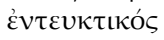

हैข

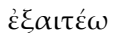

$\dot{\varepsilon} \xi \alpha \lambda \lambda \alpha \dot{\alpha} \sigma \sigma \omega$

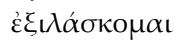

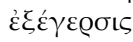

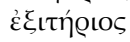

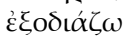

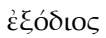

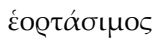

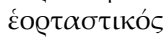

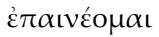

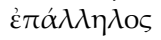

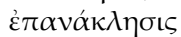

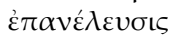

ह̌ं $\alpha \varrho \sigma \iota \varsigma$

ह้ $\pi \alpha v \lambda \mathrm{s}$

غ่тท́

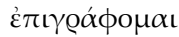

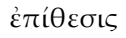

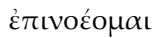

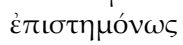

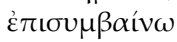

غ̇ंí́ $\alpha \gamma \mu \alpha$

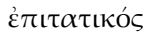

2,18

8, 2

2,5

14,3

10,7

14,3

2,13

9,28

7,16

4,12

9,9

9,2

6,28

13,3

7,15

7,14

4,23

4,24

11, 14

10,3

4,18

9,5

4,21

14,5

5,2; 11,33

13,11

11,15

14,20

7,17

6,26

14,15

4,3

7,14

$\mathbf{9 , 2 2}$

$\mathbf{9 , 1 9}$

6,26

5,5

9,2,4

8,11

5,19

10,27

11,18

14,5

14, 18

9,3

7,26

7,8

8,3

8,12

12,15

12,3

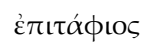

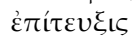

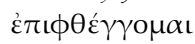

غ̇ $\pi \dot{\phi} \phi \theta \varepsilon \gamma \mu \alpha$

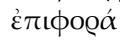

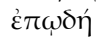

غ̇อóoutos

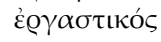

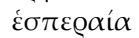

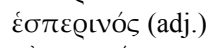

$\varepsilon \dot{\alpha} \alpha \varrho \varepsilon \sigma \tau \varepsilon \dot{\varepsilon} \omega$

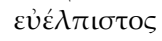

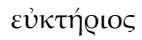

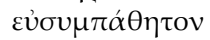

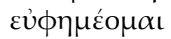

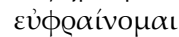

$\varepsilon \dot{v} \omega \chi i ́ \alpha$

غ̇фó $\mu \iota \lambda \lambda \circ$

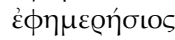

$\dot{\varepsilon} \phi \cup \beta \rho i ́ \zeta \omega$

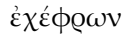

$\tilde{\varepsilon} \omega \theta \varepsilon \nu$

$\zeta \omega \mathrm{\sigma} \pi \lambda \alpha \dot{\sigma} \tau\rceil \varsigma$

$\eta \dot{\lambda \iota \kappa \iota \omega ́ \tau \eta ฺ ~}$

$\theta \varepsilon \varrho \alpha \pi \alpha$ เvís

$\theta \varepsilon \omega ́ \emptyset \eta \mu \alpha$

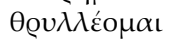

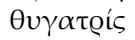

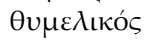

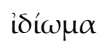

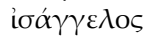

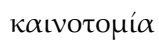

$\kappa \alpha \lambda \lambda \iota \mu \eta \dot{\tau \omega \varrho}$

$\kappa \alpha \varrho \delta \mathrm{\iota} \alpha \lambda \gamma \varepsilon \dot{0} \mu \alpha \mathrm{\iota}$

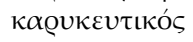

$\kappa \alpha \tau \alpha \dot{\lambda} \nu \mu \alpha$

$\kappa \alpha \tau \alpha \mu \alpha \lambda \alpha \kappa i \zeta о \mu \alpha \iota$

$\kappa \alpha \tau \alpha \pi \alpha \tau \varepsilon \dot{\omega} \omega$

$\kappa \alpha \tau \alpha \varrho \tau i \zeta$ о $\mu \alpha \iota$

$\kappa \alpha \tau \alpha \varrho \tau i \zeta \zeta \omega$

$\kappa \alpha \tau \alpha \sigma \phi \varrho \alpha \gamma i \zeta \omega$

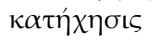

$\kappa \alpha \tau о \pi \tau \varepsilon \dot{\omega} \omega$

$\kappa \varepsilon \lambda \lambda \iota \omega \tau \iota \kappa \tilde{\omega} \varsigma$

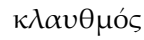

$\kappa \lambda \alpha v \theta \mu v \rho \leftarrow \kappa \tilde{\omega} \varsigma$

$\kappa \lambda \alpha v \theta \mu v \dot{\varrho} \leftarrow \sigma \mu \alpha$
Tit.

11,18

13,13

7,20

8,11

2,13

14,3

11,30

9,15

11,17

14,23

1,6

13,14

10,10

5,34

14,9

3,16

7,24

$\mathbf{5 , 4}$

5,11

$\mathbf{9 , 2 3}$

9,18

1,3

6,14

4,8

14,3

8,13

8,5

3,17

14, 11

7,2

6,9

2,6

7,9

5,6

9,10

7,18

6,17

14,22

4,20

13, 14

Tit.; 1,13

14, 6

8, 4

9,21

9,10

7,20 


\begin{tabular}{|c|c|c|c|}
\hline 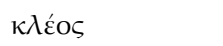 & 4,15 & 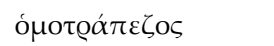 & $\mathbf{5}, 31$ \\
\hline$\kappa \lambda \eta \delta o v ı \sigma \mu o ́ \varsigma$ & 2,12 & ỏ@Ө@ıvós & $\mathbf{1 1}, 17$ \\
\hline 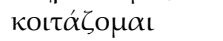 & 4,$30 ; 14,21$ & ọ́veov & $\mathbf{5 , 6}$ \\
\hline 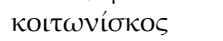 & $\mathbf{2}, 13 ; \mathbf{5}, 15$ & öభov & 5,5 \\
\hline кó@tov & 4,26 & & \\
\hline 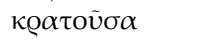 & 10,28 & $\pi \alpha \mathrm{t} \delta \alpha \gamma \omega \gamma \varepsilon \dot{\varepsilon} \omega$ & 14,16 \\
\hline$\kappa \varrho \alpha \tau \tilde{\omega} \nu$ & 10,16 & 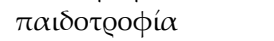 & 4,27 \\
\hline$\kappa \varrho \varepsilon о \phi \alpha \gamma \varepsilon \dot{\varepsilon} \omega$ & 3,15 & $\tau \alpha \lambda \iota v \sigma \tau \varrho o \phi \varepsilon ́ \omega$ & 9,13 \\
\hline 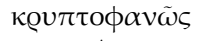 & 9,15 & 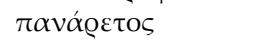 & 4,17 \\
\hline \multirow[t]{2}{*}{ 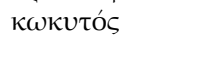 } & 8,19 & $\pi \alpha \varrho \alpha i ́ v \varepsilon \sigma \iota \varsigma$ & $\mathbf{4 , 1 8}$ \\
\hline & & $\pi \alpha \varrho \alpha \lambda v \pi \varepsilon \dot{\varepsilon} \omega$ & 3,5 \\
\hline 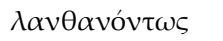 & 9,7 & $\pi \alpha \varrho \alpha \pi \circ \lambda \alpha u ́ \omega$ & 4,16 \\
\hline 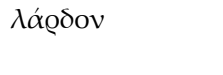 & 5,4 & $\pi \alpha \varrho \alpha ́ \sigma \tau \alpha \sigma \iota \varsigma$ & $\begin{array}{l}11,16 ; 12,6 \\
14,26\end{array}$ \\
\hline$\mu \alpha \kappa \kappa \alpha \beta \alpha \ddot{\prime} \kappa o ́ \varsigma$ & 6,21 & 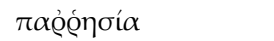 & $\mathbf{7 , 7} ; \mathbf{1 4}, 14$ \\
\hline$\mu \varepsilon \gamma \alpha \lambda$ oßov́ $\lambda \omega \varsigma$ & 6,19 & $\pi \varepsilon \lambda \alpha \dot{\zeta} \zeta \mathrm{o} \alpha \mathrm{\iota}$ & 8,10 \\
\hline$\mu \varepsilon \gamma \alpha \lambda o \psi v \chi \chi_{i}^{\prime} \alpha$ & 9,29 & $\pi \varepsilon @ i ́ \alpha \mu \mu \alpha$ & 2,12 \\
\hline$\mu \varepsilon \theta \eta \lambda \iota \kappa i ́ \omega \sigma \iota \varsigma$ & 2,4 & 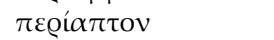 & 2,14 \\
\hline$\mu \varepsilon \theta$ o@í̧о $\mu \alpha \iota$ & 14,5 & 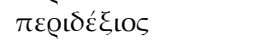 & 4,21 \\
\hline$\mu \varepsilon \sigma \eta \lambda \iota \kappa \iota o ́ \omega$ & 6,13 & 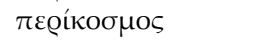 & 7,3 \\
\hline 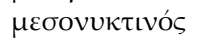 & 11,17 & $\pi \varepsilon \varrho ı \pi \lambda$ оки́ & 7,15 \\
\hline$\mu \varepsilon \tau \alpha \gamma \omega \gamma \eta \dot{~}$ & 8,11 & 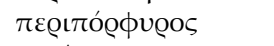 & 4,21 \\
\hline 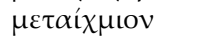 & 9,11 & 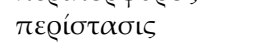 & 8,13 \\
\hline 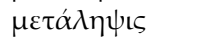 & 5,7 & $\pi \varepsilon \varrho ı ⿻ \alpha \chi \eta ́ \lambda ı \nu$ & 2,13 \\
\hline 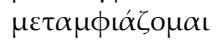 & 7,3 & 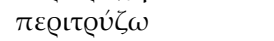 & 10,5 \\
\hline$\mu \varepsilon \tau \alpha v \alpha ́ \sigma \tau \alpha \sigma \iota \varsigma$ & 6,10 & $\pi \varepsilon \rho \iota \chi \alpha \varrho \tilde{\omega} \varsigma$ & 9,10 \\
\hline$\mu \varepsilon \tau \alpha \chi \omega \varrho \varepsilon ́ \omega$ & 1,$4 ; 14,4$ & $\pi \tilde{\eta} \xi 1 \zeta$ & 11,16 \\
\hline 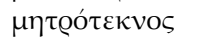 & 14,3 & $\pi \lambda \eta \sigma \mu о v \eta ́$ & 13,6 \\
\hline 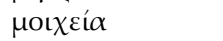 & 8,13 & 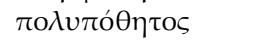 & 14,1 \\
\hline$\mu о \iota \chi \varepsilon v ́ \omega$ & 8,22 & $\pi \varrho о \alpha ́ \gamma о \mu \alpha \iota$ & 2,2 \\
\hline$\mu o v \varepsilon ́ \mathrm{o} \mu \alpha \mathrm{\iota}$ & 8,17 & 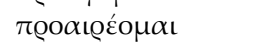 & $\mathbf{8}, 21$ \\
\hline 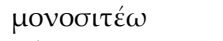 & 13,4 & $\pi \varrho 0 \ddot{\varepsilon} \mu \alpha \iota$ & 8,18 \\
\hline 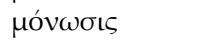 & 11,25 & 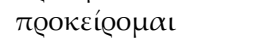 & 8,5 \\
\hline$\mu v \sigma \tau \alpha \gamma \omega \gamma \delta$ ś & 2,18 & 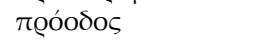 & 11,27 \\
\hline \multirow[t]{2}{*}{$\mu \omega \mu \varepsilon \varepsilon_{0} \mu \alpha \mathrm{t}$} & 12,2 & 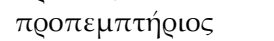 & 7,14 \\
\hline & & 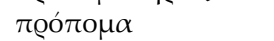 & $\mathbf{5 , 6}$ \\
\hline veóүovos & 2,11 & $\pi \varrho 0 \sigma \delta \varepsilon ́ \chi 0 \mu \alpha \iota$ & 10,6 \\
\hline 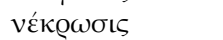 & 9,25 & $\pi \varrho о \sigma \varepsilon \pi \varepsilon \mu \beta \alpha i ́ v \omega$ & $\mathbf{8 , 2 8}$ \\
\hline vođع@ós & 5,29 & $\pi \varrho о \sigma \varepsilon \pi \iota \tau i ́ \theta \varepsilon \mu \alpha \iota$ & 9,20 \\
\hline 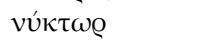 & 11,29 & $\pi \varrho о \sigma \varepsilon \varrho \varepsilon i ́ \delta о \mu \alpha \iota$ & 11,2 \\
\hline 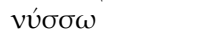 & 4,32 & 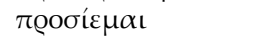 & 8,22 \\
\hline \multirow[t]{2}{*}{$v v \sigma \tau \alpha \dot{\zeta} \zeta \omega$} & 12,7 & 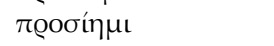 & 11,8 \\
\hline & & $\pi \varrho о \sigma о \mu \iota \lambda \varepsilon ́ \omega$ & 7,7 \\
\hline \multirow[t]{2}{*}{$\xi \varepsilon v o \delta o \chi \varepsilon ́ \omega$} & 6,3 & 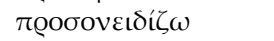 & 8,28 \\
\hline & & 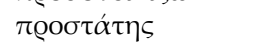 & 10,8 \\
\hline 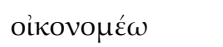 & 10,24 & $\pi \varrho о \sigma \tau u ́ \pi \tau \omega$ & $\mathbf{5}, 11$ \\
\hline oùкovó & 10,8 & 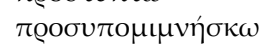 & $\mathbf{1 4}, 19$ \\
\hline 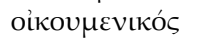 & 8,15 & $\pi \varrho o ́ \sigma \phi \alpha \tau о \varsigma$ & 5,5 \\
\hline oikcov@í $\alpha$ & 4,5 & $\pi \varrho о \tau \dot{\varrho} \emptyset \mu \alpha$ & $\mathbf{5 , 8}$ \\
\hline ó $\mu \varepsilon \dot{\varrho} о \mu \alpha \mathrm{t}$ & 5,2 & 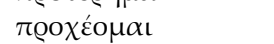 & 11,3 \\
\hline 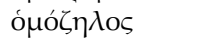 & 10,9 & 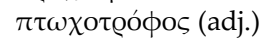 & $\mathbf{5 , 3 0}$ \\
\hline 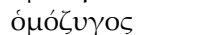 & 4,7 & & \\
\hline
\end{tabular}




\begin{tabular}{|c|c|c|c|}
\hline 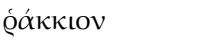 & 13,9 & 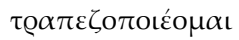 & 11,11 \\
\hline @́ $\alpha \pi \hat{\imath} \zeta \omega$ & $\mathbf{5 , 1 1 ; \mathbf { 1 2 , 7 }}$ & 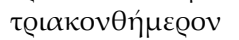 & 10,17 \\
\hline 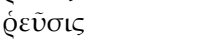 & 14,27 & tQíxıvos & 13,9 \\
\hline \multirow{2}{*}{ @v $v \mu$ óo $\mu \alpha \iota$} & 14,22 & 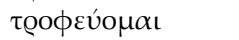 & 10,7 \\
\hline & & 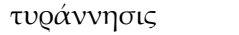 & $\mathbf{3}, 20$ \\
\hline$\sigma \alpha \mu o v \eta \lambda i ́ \tau ı \varsigma$ & 6,22 & & \\
\hline$\sigma \eta \mu \varepsilon เ o ́ o \mu \alpha \iota$ & 4,30 & $\dot{v} \pi \alpha \lambda \varepsilon \dot{i} \phi \omega$ & 9,$8 ; 10,6$ \\
\hline 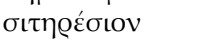 & 5,4 & & 11,27 \\
\hline$\sigma \kappa \varepsilon ́ \tau \alpha \sigma \mu \alpha$ & 13,10 & $\dot{v} \pi \alpha v \alpha \gamma \kappa \alpha \dot{\zeta} \zeta \omega$ & 4,24 \\
\hline 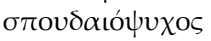 & $\mathbf{5 , 1 3}$ & $\dot{v} \pi \varepsilon \lambda \alpha \tau \tau o ́ \omega$ & 3,5 \\
\hline$\sigma \tau \varepsilon ́ v \omega \sigma t \varsigma$ & 11,8 & $\dot{v} \pi \varepsilon \xi \dot{\alpha} \gamma \omega$ & 10,7 \\
\hline$\sigma \tau \varepsilon \dot{\varrho} \gamma о \mu \alpha \iota$ & 5,21 & 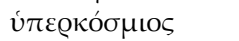 & 1,4 \\
\hline 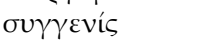 & 6,$11 ; 8,5$ & 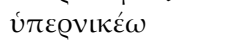 & $\mathbf{7 , 2 1 ; \mathbf { 1 4 } , 7}$ \\
\hline$\sigma v \gamma \gamma \varrho \alpha ́ \phi \omega$ & 2,2 & 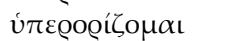 & $\mathbf{9}, 14 ; \mathbf{1 0}, 26$ \\
\hline$\sigma v \gamma \kappa \alpha \theta \varepsilon v ́ \delta \omega$ & 4,14 & 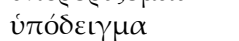 & 4,7 \\
\hline 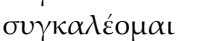 & 11,12 & 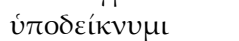 & $\mathbf{4}, 22 ; \mathbf{5}, 22$ \\
\hline 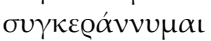 & 9,23 & v́тó $\mu \nu \eta \mu \alpha$ & 4,27 \\
\hline 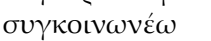 & 14,11 & 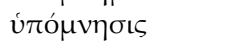 & 4,8 \\
\hline 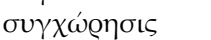 & 12,11 & 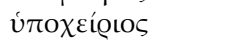 & 8,28 \\
\hline$\sigma v \gamma \chi \omega \varrho \iota \alpha ́ \zeta \omega$ & 14,6 & & \\
\hline 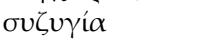 & 6,12 & $\phi \iota \lambda \varepsilon \varrho \gamma^{\prime} \alpha$ & 11,29 \\
\hline 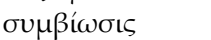 & 2,$5 ; 14,12$ & фuגótovos & 4,5 \\
\hline$\sigma v \mu \mu \varepsilon \tau \varrho t \alpha ́ \zeta \omega$ & 5,24 & $\phi \iota \lambda о \pi \tau \omega \chi \underline{i} \alpha$ & 11,13 \\
\hline$\sigma v \mu \mu i ́ \gamma \omega$ & 12,17 & $\phi о \beta о \theta \varepsilon \ddot{\imath} \alpha$ & 9,28 \\
\hline 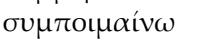 & 14,19 & 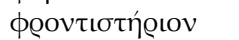 & 10,3 \\
\hline 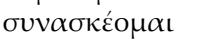 & $\mathbf{1 0}, 18 ; \mathbf{1 2 , 4}$ & ф@ov@ev́s & $\mathbf{1 0}, 19$ \\
\hline 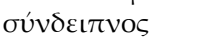 & 5,31 & $\phi \omega \tau \alpha \gamma \omega \gamma \varepsilon \dot{\varepsilon} \omega$ & 3,6 \\
\hline$\sigma v \vee \varepsilon \kappa \delta \eta \mu \varepsilon ́ \omega$ & 8,25 & & \\
\hline$\sigma v v \varepsilon ́ \lambda \kappa о \mu \alpha \mathrm{\iota}$ & 6,4 & $\chi \alpha \mu \alpha i ́ \zeta \eta \lambda$ os & 4,25 \\
\hline$\sigma u v \theta \varrho \alpha u ́ \omega$ & 8,25 & $\chi \varepsilon\llcorner\varrho \alpha \gamma \omega \gamma \varepsilon \dot{\epsilon} \omega$ & 8,6 \\
\hline 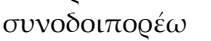 & 8,25 & 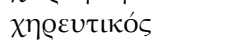 & 3,13 \\
\hline 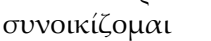 & 11,6 & $\chi \ominus \alpha \mu \alpha \lambda o ́ \varsigma$ & 8,24 \\
\hline 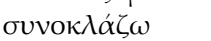 & 7,19 & 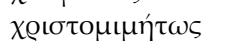 & 11,11 \\
\hline 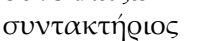 & 9,19 & $\chi \omega \mathrm{Q} \alpha$ ко́ & 9,15 \\
\hline$\sigma \chi \varepsilon ́ \delta ı \varsigma$ & 13,5 & & \\
\hline$\sigma \chi 0 \lambda \alpha ́ \zeta \omega$ & 3,4 & $\psi \alpha \lambda \mu \omega \delta \delta^{\prime} \alpha$ & 12,6 \\
\hline 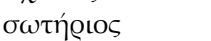 & 13,14 & $\psi \alpha \lambda \tau$ tท́อเо & 3,3 \\
\hline & & 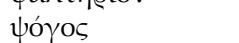 & $\mathbf{5}, 11$ \\
\hline 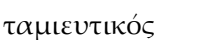 & 6,14 & & \\
\hline
\end{tabular}




\section{Translation (J. M. Featherstone)}

\section{Of Our Holy Father and Confessor Theodore: Funerary Catechism for His Mother}

1. I must now announce to you, my children and brothers, the unexpected news of the death of the glorious sister of our common father: for by the fixed counsel of God, the Creator of life, after having ministered in this present life she has passed over into the celestial world. She has not left us with grief beyond reason, as one might think, but has instilled in us joy through her departure in good hope. Would you like me to tell you and all those present here (for it is now the time not to keep silence but to speak, ${ }^{41}$ in accordance with the commandment which enjoins: Thou shalt not praise any man until his passing) of all the good and pious things, worthy of the kingdom of heaven, done by this blessed woman whilst she was alive? Then lend me your ears, my children, and I shall narrate everything to you, removed from all falsehood; and this narration concerning her shall be unto you even as an instruction in faith, nor one lacking in reason, but indeed most beneficial.

2. That her virtue was derived, as her birth, from her parents, I cannot say, for it is not my purpose to write about things before my time, but only afterwards, from the time when she had gained experience in the judgement of that which was good and that which was not. Let us then pass over the first and second periods of her life and begin our narrative with the time of our communal life. ${ }^{42}$ Even if this glorious mother's secular life is not a subject familiar to us, nevertheless, in order that we may see the end from the beginning, I shall of necessity add this. Her first achievement, then, was to revere and love God wholly, so that even if she was married to a man, still she strove toward the higher power. For this reason, at first, when she gave birth to us, she did not follow the example of other women, accustomed as they are, through diabolic impulse, to use charms and amulets and other enchantments on their newborn children in their seats and rooms, hanging necklaces and talismans upon them, but she was satisfied simply by sealing us with the sign of the life-giving cross, using this instead of any other arm or irresistible armour. ${ }^{43}$ Thus, whereas all other women worshipped and showed deference in the presence of the author and guide and teacher of such things, our mother was

41 As a rhetorical formula this saying opens ep. 439, ed. Fatouros, 617; see apparatus fontium (ibid.) for other parallels from the Stoudite's æeuvre.

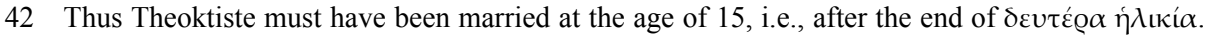
Theodoros' year of birth (759) points to a birth date in ca. 743/744 at the latest. On the reckon-

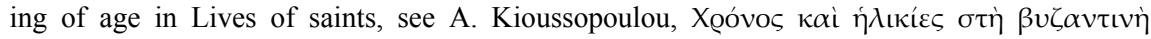

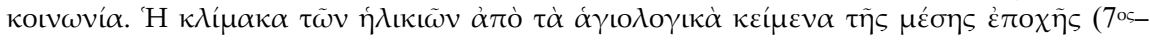

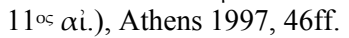

43 For the association of women with superstitious practices perpetuating pre-Christian beliefs see J. Herrin, In Search of Byzantine Women, in: Images of Women in Antiquity, ed. Av. Cameron/A. Kuhrt, London ${ }^{2} 1993$, 172. For the association of these practices with birth see Ph.

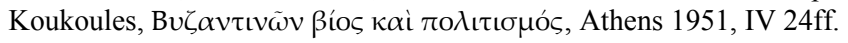


alone in her refusal, neither bowing her head nor approaching nor taking part in any sorcery, though she was often threatened by those who performed such things. But verily, the souls of the righteous are in the hand of God and no torment shall touch them; and He that trusts in the Lord shall be as Mount Zion, as Scripture saith.

3. Later, when divine longing had increased in her heart, because she was illiterate in her orphan state, this wise one instructed and taught herself, and she learnt the Psalter by heart in best and quickest fashion. How did she do this? Not by occupying herself with study during the day, lest she should annoy her husband or neglect the household, but before bed-time and after bed-time, working with effort by the light of a lamp; nor did she neglect her manual work. But attending now to this latter, and now following the other pursuit, she brought increase to the household and acquired learning. Thereafter she ceased not conversing day after day with the divine David and gave heed to the divine readings; and, thereby growing fervent in heart and striving brightly for the bounty to come, what did this woman worthy of wonder do and what was it she wrought? Divesting herself of vain things and putting on a sort of widow's attire, she set herself as an example of good to those who knew her, swearing naught whatsoever, nor telling falsehood, nor, for the most part, eating any meat, especially in Lent. Even when invited to a marriage feast she secretly contrived not to eat any meat nor to raise her eyes to the theatrical performances. For she was prudent as any woman ever was, knowing but one man and, after renouncing the world, keeping her soul so pure as to impose rigour to the extent of absolute mastery over her thoughts.

4. When did she ever miss the midnight office? For she put into practice the saying: At midnight I will rise to give thanks unto Thee because of Thy righteous judgements. When did she not rise for Matins? Or did she miss out any other set hour of thanksgiving? In no wise! But rather, applying herself to the running of the household with rigour and no common assiduity she expended most ample effort in good deeds and, through the example of herself, affected the life of her husband and her children and her maidservants. As regards her husband, amongst other things, she brought him to the remembrance of the separation of death and, taking counsel with him, sought that they should abstain from one another. Thereby she convinced him, and they slept in one bed for all of five years or more without knowing one another: a most strange and unheard-of thing! For how could fire not converse with straw lying beside it, unless by extraordinary occurrence? If Boaz be praised for leaving Ruth untouched as she slept beside him one night, how can these two not rightly win renown for their prudence? This is praise for both of them: for he who begat reaped here no inopportune advantage from the praise for her who bore. This all-virtuous mother educated the children ${ }^{44}$ with counsels and exhortations, now striking them with the rod of reason, now leading them with care, conducting them and bringing them to increase in divine things.

44 Mai added $\tau \dot{\varepsilon} \kappa v \alpha$ after $\tau \dot{\alpha} \delta \varepsilon \dot{z}$; but, in view of the preceding $\tau o ̀ v \mu \varepsilon \dot{v}$, this is unnecessary. 
What then were the results? She formed the daughter in a marvellous way: she never brought her into the sight of males, nor gave her the example of feminine tresses or bracelets or purple garments, but brought her up in piety and instructed her in sacred Scripture, teaching her to receive the poor and requiring that she treat their leprous sores with her own hands. In sum, leading the girl's mind away from the lowly things of this earth toward God and the beauties of heaven she consecrated her to God. ${ }^{45}$ But although the topic of her excellent rearing of children is replete with a great many notes in my memory, let us stop here for fear of surfeit. Let me add only one last thing which I think ought not to be omitted: that after she had put the children to bed, she would never herself go to bed before making the sign of the cross over their limbs as she went out; and again, when she rose she most often roused and woke us and exhorted us to prayer, so that not only she but also we children learned to worship God.

5. There is much that I should like to say about how she took care ${ }^{46}$ for the servants, both male and female; she nourished them with food and drink and clothed them as no one else, not even the most benevolent of masters, distributing bread and wine and lard in no ordinary wise as daily rations but in abundant fashion; and on feast days she also offered fresh meat, ${ }^{47}$ fish and poultry and seasoned drinks, for she could not bear to keep the enjoyment of such things for herself. Were these excellent traits of this holy soul not admirable and astonishing? I know that you who are listening will agree. But in addition I should also say that she would utter warnings and admonitions, and in the case of those around her - I do not know whether to cite this as praise or reproach - she would give thrashings and scoldings and beatings in order to insure prudence and honesty ${ }^{48}$ and other virtue, her earnest soul being filled with anger, for she was of a rather sharp nature, often afflicted through her zeal for God. But it was also her way, after she had struck someone, to retire to her room and strike herself on the cheeks: 'Let that smart!' she would say to herself, and thereby brought herself to repentance; and summoning the maidservant she had struck and kneeling down she would ask forgiveness. Oh, what pious recall to reason! For though one might reproach the former deed, one would nevertheless praise the second so marvellously accomplished. Wherefore, mixing fear with mercy, she came to be loved by her maidservants and set a good example to them of the observance of prudence; and she gained the high ground in both respects. Who surpassed her in being loved by those around her or in almsgiving to the needy? The one she obtained by her evenly measured treatment, especially towards inferiors, and the other by her

45 For other examples of a "monastic" upbringing of younger daughters as a preparatory step to their final dedication to a convent see de F. Abrahamse, Women's Monasticism (see n. 21), 49 50.

46 The rare verb ó $\mu \varepsilon$ íoo $\mu \alpha \mathrm{\iota}$ is also used in epp. 7,17 and 438,12, ed. Fatouros, 24 and 616 respectively.

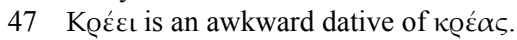

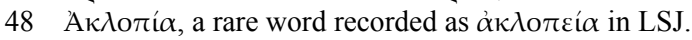


great sympathy, even if the moderate nature of her property did not permit her to display the exuberance of her purpose. But witnesses to the truth were many, mostly and particularly the orphans and widows, strangers and natives, the sick and the old, and those afflicted with leprosy, who were provided for by her generous right hand. And this was also a singular trait of hers: that she had Christ as a companion at table, especially at the time of feasts. These things seem to me of no small import as provision for the salvation of those in married state, even if we have yet greater things to say concerning her who is the subject of our praise. ${ }^{49}$

6. Now, when the time came for her calling to the monastic habit, who before her so received and gave shelter and served the servants of Christ, being prompted in this by her own brother? Thus it was - I pass over the details - that out of her own desire for the ascetic life she persuaded her husband, and she persuaded also her children and even her husband's brothers, with many efforts and counsels and promises. ${ }^{50}$ Arranging everything in good order, she left the house, committing to God the entire household: the four children, three brothers-in-law and herself and her husband. Oh, what a wonderful innovation! What an unexpected migration! This event took the empress by surprise on the very day; it astonished the relations of the family; it left acquaintances perplexed; it bewildered not only those who saw it but also those who heard: that a married couple still in middle age and self-sufficient in their livelihood, holding an imperial dignity in the treasury and having grown children was not bound by affection for these latter, nor desire for the succession of their race, nor the bonds of kinship, nor the alienation of their household, nor yet the loss of their servants; but rather they cast away imperial dignities, they trampled upon the delights of this life, with the sword of the spirit they cut themselves off from fleshly relations and magnanimously did that which was pleasing to God. Oh most valiant souls, verily worthy of Abraham! Rightly might one place them together with that excellent mother of the Maccabees who offered the product of her womb to ascetic practices, and with Anna the mother of Samuel who consecrated to the Lord not only the one son she promised but all of the fruit she bore. But I shall regale you listeners with something perhaps even more pious by narrating the manner of her withdrawal. On the day she had set to leave the house, as if it was a festal day, she invited all her relations. The men were aggrieved, and the women wailed beholding the spectacle of this voluntary separation, though, considering within themselves the greatness of this mystery, they praised the deed. For all observed how the husband left the house, and the wife, remaining but a while, then went off to the monastery; and the house itself

49 As the orator is about to speak of the monastic feasts of his mother, the oúk of the MS should rather be put between obels.

50 This "willy-nilly" embracing of the monastic life must have occurred in 782-783. Theoktiste's husband, his three brothers and his three sons became monks in Sakkoudion, founded by Platon on a family estate called Boskytion. Cf. Laudatio Platonis, in: PG 99, col. 824B-C and vita A of Theodoros Stoudites, ibid., col. 121D, and vita B, ibid., col. 241B. 
was sold and the proceeds distributed to the needy. These are the achievements of our mother's secular life. I have recounted them so that not only you, but whosoever shall come into this world later shall be edified by these most excellent narratives and shall give thanks to God.

7. Come, then, beloved, let us look to what happened next: this is the main subject of our speech. This most decorous woman made the good change, vesting herself in the angelic habit. She moved to tears the many who had assembled on the day of this event, some of them unbidden, in order just to see the enactment of this mystery. We were also there, together with our common father, in order - should I say, to celebrate, or to wail? For having lost our mother, never again to speak nor to approach her with the same boldness, and contemplating our separation from her, we were grieved at heart. And when, in the fullness of time, we too, together with our father, were to go off and be consecrated, I, having barely reached the state of manhood, on the one hand bore this experience with pain and grief - how could it not be so? -, but on the other with thanksgiving. The younger of my two brothers, however, being very young indeed, on the day of our departure, after all the speeches of leave-taking and farewell, the invocations and lamentations and embraces, ran up and hugged our mother, clinging to her desperately, even as a calf rejects being weaned, and begged to be allowed to remain with her a bit longer, promising to fulfil her desire later. What then? Was that adamantine heart softened? Did she agree, or cede before the weeping of her child? In no wise! What was her pious response? With a fierce expression she prevailed over her strong feelings of motherly affection: 'If you do not go willingly, child, I shall put you into the boat with my own hands.' The boy yielded; and we both went off. As for our mother, it seems to me that she here accomplished a deed equal to that of the holy mother at the martyrdom of the Forty who lifted her own son who was still alive and placed him in the cart. ${ }^{51}$

8. What happened next? This noble woman desired to live under a rule, as was fitting. But since, on account of the earlier turbulence there was no monastery in existence, nor yet any soul capable of knowledgeably leading the many, for this reason she was obliged by her brother to live amongst cell-dwellers together with her daughter who had already been tonsured and another female relation. ${ }^{52}$ Straightaway she began a life of patience, but also one of distress ${ }^{53}$ - how could it not be so? -, seeing that she had acquired no guide and, what is more, discovered women who acted in opposition to piety. It came to such a pitch that she was expelled from the holy place - I am ashamed to say, in consideration for the lis-

51 Reference to the mother of the last survivor of the 40 Martyrs of Sebastia, who placed her son on the cart bound for the fire so that he could not avoid martyrdom; see St Basil's Homily 19 (BHG 1205), in: PG 31, col 524B-C. Commentary by P. Karlin-Hayter, Passio of the XL Martyrs of Sebasteia. The Greek tradition: the earliest account (BHG 1201), in: AB 109 (1991) 284 287.

52 Theoktiste must have dwelt in a separate cell not far from Sakkoudion.

53 Note the consecutive use of adverbs gradually leading from a better to worse condition. 
teners, why or by whom; but she bore up, suffering on behalf of good and sojourning for the sake of the Lord. I know not how to tell of the vicissitudes and shiftings back and forth of her life, nor of the afflictions and pains and perils which befell us, both from our relations and from the imperial power. The culmination was the notorious adultery of the emperor, on account of which our mother also suffered. Now, since I have mentioned this shameless act of universal dimension, you should know how hard it was for her when, being present at the time, she saw us being taken away from the monastery. But even if she was especially grieved at being bereft of us, she was not vexed, she pronounced no ignoble word, she rent not her garment nor raised any shriek. 'Go forth,' she said, 'my children and be saved in the Lord. Whithersoever you may go and whatsoever you may suffer, you choose this on account of His law. For it is good that you should suffer any evil even unto blood rather than to take the part of the adulterer and thereby betray the truth.' O valiant and noble soul which did not forthwith cede, as one might expect, to lowly and cowardly passion! What then? She also departed, she also made the journey, breaking her delicate body in passing over the mountain; nor did she find anyone willing to make the trip with her. Alas! How inhuman had all men become at the time! Some of her servants even came up to her and insulted her, thus fulfilling the saying of the Gospel: And a man's foes shall be they of his household.

9. Thus this blessed woman anticipated us in our suffering. Now, let no one think that we are here praising ourselves, we who have nothing worthy of praise; for it was others, not we, who acted courageously, and the deed is to be ascribed to our common father. But in order that we might narrate the struggle of her who is the subject of praise, we have been brought, - forgive us! - unwillingly, to the use of these words concerning ourselves. But Great is our Lord, and of great power. As she secretly stole into the prison and saw the wounds from the beatings and embraced them, anointing them with salve, she sent up glory and praise to God that her offspring had suffered these things for the sake of His commandment. Thus in a prayerful and weeping but also exceedingly glad manner, in the midst of these two feelings, did she accompany us in the removal from one place to the next, deemed blessed by God and men of pious disposition. Thus did she come to us on our return from Kathara, ${ }^{54}$ on the way to exile in Salonica, one wintry evening, in a rustic inn, secretly and in disguise for fear she should be seen, and she passed nearly the whole night with us. ${ }^{55}$ Having made covenants with one another in the

54 The latest discussion of the location of Ta Kathara is by Cheynet/Flusin, Du monastère Ta

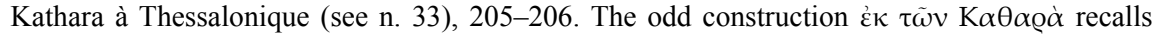
$\dot{\alpha} \pi \dot{\mathrm{o}} \tau \dot{\alpha} \mathrm{K} \alpha \theta \alpha \varrho \dot{\alpha}$ as in ep. 3,68. Though $\mathrm{K} \alpha \theta \alpha \varrho \dot{\alpha}$ is accented with a circumflex by Fatouros, Theodori Studitae epistulae (see n. 3), 13 and Cheynet/Flusin, Du monastère Ta Kathara à Thessalonique (see n. 33), 198, in all extant manuscripts the word is accented with a grave.

55 Theodoros refers to this secret meeting in ep. 3,75-6 to Plato: see Theodori Studitae epistulae (see n. 3), 14. In this letter he gives a detailed account of all the intermediary stopping-off points from Kathara to Thessalonike. See Cheynet/Flusin, Du monastère Ta Kathara à Thessalonique 
Lord, at dawn - Oh, how unhappy and painful it was! - we were forthwith to be parted from one another; and bidding us farewell, as a final gesture she kissed us on each of the limbs of our bodies with weeping. 'Now, my children,' to cite her very words, 'it seems to me that I address you for the last time, for to the prudent is given the power of conjecture. If it is with those who are commingled in love toward one another to be parted, according to the Gregory the great Theologian, even so as the cutting of one body into two and the mortification of both, or as the separation of calves bred and yoked together which bellow mournfully and cannot bear their estrangement, what are we to do in the present circumstances, even if we overcome our feelings of pain through fear of God?' Let this great example of her high-mindedness be praised.

10. As for what followed, brethren, you may narrate yourselves, you who benefited from her good deeds: how, setting out for her journey to us, she gathered you, like nestlings thrown from the nest, into her own monastery; then, returning, how she comforted each one whilst making her way into the city. For having entered somehow therein, with sighing and supplication, she received one with hospitality and assuaged another's pain with salves; some she propped up, and others she secretly led away; some she nourished, others she provided for, even as an excellent steward and godly protector, in verity a new Natalia ${ }^{56}$, an emulator of Priscilla's zeal ${ }^{57}$. You are witnesses thereto, you who have profited and who foster the oral account and praise of her beneficence. But then this admirable woman was deemed worthy of the final blessing when she was reproached by certain men, whose names I willingly omit, for she was persecuted for righteousness' sake and truth. Answer me yourself, father: what did this much-labouring woman bear for your sake in reproach, in disputation and in solicitude for necessities whilst you were detained in prison? Tell of the ruler's plundering, the threats, the investigations. Did she not suffer together with you, remaining thirty days incarcerated with four companions in ascetic practice, all being kept apart in prisons? I omit to tell of all she suffered from the maidservants of the gaoler, the hardship she endured with regard to the necessities of life, eating the bread of pain and drinking a draught of affliction. But this is not the time to talk about the things that were inflicted with impudence and occurred then. Let us forgive the perpetrators whatever they did. For it is more fitting to speak thus. Whether by His judgements God allowed or else disposed that evil should take hold and the unrighteous should not submit to the law, it was He who called forth the persecu-

(see n. 33), 201-211. These authors considered that the mother and son's meeting occurred on 20 February 797 at Paula, situated ca. $20 \mathrm{~km}$ east from Lopadion. For this town see R. Bondoux, Les villes, in: La Bithynie au Moyen Âge, ed. B. Geyer/J. Lefort (Réalités byzantines 9), Paris 2003, 392-394.

56 St Natalia was the wife of St Adrianos and fervent supporter to his sudden decision to renounce his pagan belief and join the other Nicomedian martyrs who suffered under Maximian; see their Passio BHG 27, AASS Sep. III, p. 218-230.

57 Apart from ch. 18 of the Acts, Priscilla is mentioned in Rom. 16,3; along with her husband Aquilas, she was much involved in St Paul's mission to the Gentiles. 
tion; and we who had been exiled and dispersed then came back together, having obtained our happy return from the empress.

11. But what can we offer to this God-honouring mother in addition to what has already been said? What can we say that is yet more fitting? O streams of tears which forever flowed from her eyes for her husband, for the least stalwart of her children, for her flock, for those who fell upon the slippery path, for the support of everyone! As a spiritual mother it was her way to have mercy and as a sister she always struggled after the manner of the Lord on behalf of her sisters, even if she did not live with them. O gift of merciful and charitable soul! O emptying of affectionate bosom unable to bear the affliction of those who approached her! so much so that she would put herself in need for the sake of those who were needy and would borrow in order to deliver others from loans; she would rejoice even to pay herself for the ransoming of others. Moreover, she would often serve at table in imitation of Christ, and inviting poor women she waited upon them with her own hands, for the gift of love for the poor was all her desire. O mouth venerable in constant study of divine Scripture and performance of the liturgy without ceasing even unto death! $O$ feet venerable in standing upright for the supplication to God with unswerving solidity and firmness! O hands holy not only in their evening, but also morning and midnight raising unto $\mathrm{God}^{58}$ and effective attainment of things besought from Him! O explanation of the dreams of night! For in the face of present grief did God often foretell her of what was to be, thus consoling His maidservant. O slackness of the whole body brought on by ascetic practice! $\mathrm{O}$ simple and insufficient ${ }^{59}$ clothing in winter! $\mathrm{O}$ bed stiff and narrow and stunted, and lying down in the cold! O unfriendly and indifferent disposition to the sight of any male! O patient and tenacious striving in solitude toward God! Wherefore, struggling against despair because she could not see us often nor had anyone else to console her spiritually, she gave way and departed on journeys and voyages, though even in this she kept a perfect rule. $\mathrm{O}$ unsurpassed love of toil by night and by day! $\mathrm{O}$ industrious hardening of the fingers, their natural softness turned to the hardness of hide, not only working to suffice for herself - for this she counted as nought! - but in order to clothe almost the whole monastery, as you who are listening know.

12. Amongst other things, she was also different from other women in the following. If one is to find fault with her, as with any human being, the reasons for this stem from her same fervour for the good, intense as she most often was in her instructions to the monastic women in obedience to her. For at times she grew angry because of faults either in the performance of tasks or psalm-singing or standing during the offices; so much so, that sometimes she could not refrain from giving a push to those who were nodding off or striking those who disobeyed. But then she could also inspire love to a greater extent than the most even-tempered

58 From Psalm 140,2 sung at Vespers.

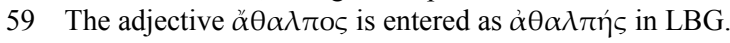


persons in so far as she was moved to these outbursts through desire for the good, and she always repented and sought to submit herself to others; and hereby she made God propitious, even as we also rightly gave pardon. For after the confinement of our father she so submitted herself and offered obedience to my humility that she called herself a maidservant and touched my unworthy feet; she revealed the secrets of her heart and showed not the slightest reluctance to any command. Wretch that I am, I am filled with shame whenever I consider how she called me lord and father and how she revered me, mixing desire with fear, and obeyed me not as a mother but as a child.

13. Moreover, she made it a rule of continence never to eat her fill, and even though obedience, to which she was very devoted, would not allow for the reduction of the quality of food, nevertheless she did not obey. She ate, therefore, but once a day, usually toward evening, partaking of some plain and ordinary food, without oil or wine; and when she broke the fast on set days she was satisfied in taking but a little, slowly, with no surfeit. It is an example of her poverty ${ }^{60}$ that she possessed neither maidservant ${ }^{61}$ nor gold nor silver nor aught of this world, save for the hair garments in which she was clothed and two covers of poor quality. When she died, she left these to me and my brother, ${ }^{62}$ keeping the rest for her burial, and thus stripped of material things and affection for this world she went home rejoicing, departing thence, addressing us with prayers and salutary words and sealing each of those who stood by with the sign of the cross, for many of the brethren were present to watch her repose.

14. $O$ venerable and much-desired mother (for I turn to you, $O$ thing and name of sweetness, beloved and longed-for sight, at once my mother and my child, or rather, my twice-born mother who bore me twice!), how could you leave us? Whither have you departed? Whither have you been taken off? Where and in what places do you dwell? In what abodes do you tarry? Whom do you now see? For having prevailed over the ruler of this air through your good deeds, I am sure that you are in that place whence pain and grief and sighing have gone away, ${ }^{63}$ where the company of all the saints rejoices, where there is song and dance of those who celebrate the feast and are joyful, and where dwell our common breth-

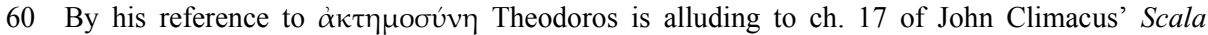
Paradisi; see S. Giovanni Climaco, Scala Paradisi, ed. P. Trevisan, Torino 1941, II 7 (= PG 88, cols 928-932).

61 Allusion anticipating a development of the early Paleologan period when monastic Typika allowed nuns of aristocratic origin to retain one or two of their servants on their entrance to the convent; see the Typikon of Theodora Paleologina for the Convent of Lips in Constantinople in H. Delehaye, Deux typica byzantins de l'époque des Paléologues, Brussels 1921, 129 (ch. 41); and the Typikon of Theodora Synadene for the Convent of the Mother of God Bebaia Elpis in Constantinople, ibid., 71 (ch. 94). Engl. transl. of both by A.-M. Talbot, in: J. Thomas/A. Constantinides Hero (eds.), Byzantine Monastic Foundation Documents (DOS XXV), Washington, D.C., 2000, 3, 1278; and ibid., vol. 4, 1550.

62 Joseph, later archbishop of Thessaloniki.

63 From the Kontakion of the funeral office. 
ren whom you loved and chose as partners in communion. For the properties of this present flesh now set at variance our communal life with you. Do not forget us, your most humble children. Do not, I pray. Do not, I entreat. Do not forget your flock, both the small and the great. ${ }^{64}$ But having obtained boldness of speech - and I believe that you have obtained it! - stand by us and propitiate and make atonement for us, pray and make supplication especially now most earnestly. Teach and uphold and guard me, wretch that I am, from sinful fear, from the flying dart of iniquity, from all other demonic harm: be as a shepherd unto me, be a shepherd together with me, prod me and remind me through prayer of the spirit. Protect me and correct me always, watching how I rise, how I lay me down, and how I am affected in soul and body, that I might be brought into harmony and perfected in every wise and, following the path of truth, might succeed in pleasing God by performing well the task of leader and might be granted, after this life, together with those who follow me, to come under your protection and to stand with you, as your most humble child, at the right hand of Christ our God.

Truth, my children, has compelled me to this narration without eloquence in response to your demand to hear these things.

64 Viz. "your" family and the Stoudite community. 
